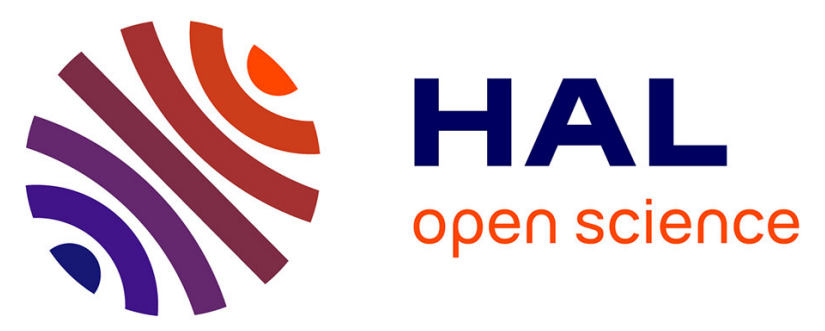

\title{
Type VI secretion TssK baseplate protein exhibits structural similarity with phage receptor-binding proteins and evolved to bind the membrane complex
}

van Son Nguyen, Laureen Logger, Silvia Spinelli, Pierre Legrand, Thi Thanh Huyen Pham, Thi Trang Nhung Trinh, Yassine Cherrak, Abdelrahim Zoued, Aline Desmyter, Eric Durand, et al.

\section{To cite this version:}

van Son Nguyen, Laureen Logger, Silvia Spinelli, Pierre Legrand, Thi Thanh Huyen Pham, et al.. Type VI secretion TssK baseplate protein exhibits structural similarity with phage receptor-binding proteins and evolved to bind the membrane complex. Nature Microbiology, 2017, 2, pp.17103. 10.1038/nmicrobiol.2017.103 . hal-01780712

\section{HAL Id: hal-01780712 \\ https://hal-amu.archives-ouvertes.fr/hal-01780712}

Submitted on 27 Apr 2018

HAL is a multi-disciplinary open access archive for the deposit and dissemination of scientific research documents, whether they are published or not. The documents may come from teaching and research institutions in France or abroad, or from public or private research centers.
L'archive ouverte pluridisciplinaire HAL, est destinée au dépôt et à la diffusion de documents scientifiques de niveau recherche, publiés ou non, émanant des établissements d'enseignement et de recherche français ou étrangers, des laboratoires publics ou privés. 


\section{Type VI secretion TssK baseplate protein exhibits structural similarities with phage}

2 receptor binding protein and evolved to bind the membrane complex

3

4 Van Son Nguyen ${ }^{1,2}$, Laureen Logger ${ }^{3}$, Silvia Spinelli ${ }^{1,2}$, Pierre Legrand ${ }^{4}$, Thi Thanh Huyen

5 Pham ${ }^{1,2,5}$, Thi Trang Nhung Trinh ${ }^{1,2,5}$, Yassine Cherrak $^{3}$, Abdelrahim Zoued ${ }^{3}$, Aline

6 Desmyter $^{1,2}$, Eric Durand ${ }^{3}$, Alain Roussel ${ }^{1,2}$, Christine Kellenberger $^{1,2}$, Eric Cascales ${ }^{3 *}$ and

7 Christian Cambillau ${ }^{1,2 *}$

$9{ }^{1}$ Architecture et Fonction des Macromolécules Biologiques, Aix-Marseille Université,

10 Campus de Luminy, Case 932, 13288 Marseille Cedex 09, France.

$11{ }^{2}$ Architecture et Fonction des Macromolécules Biologiques, Centre National de la Recherche

12 Scientifique (CNRS), Campus de Luminy, Case 932, 13288 Marseille Cedex 09, France.

$13{ }^{3}$ Laboratoire d'Ingénierie des Systèmes Macromoléculaires, Institut de Microbiologie de la

14 Méditerranée, Aix-Marseille Univ. - Centre National de la Recherche Scientifique

15 (UMR7255), 31 Chemin Joseph Aiguier, 13402 Marseille Cedex 20, France.

$16{ }^{4}$ Synchrotron Soleil, L’Orme des Merisiers, Saint-Aubin - BP 48, 91192 Gif-sur-Yvette

17 Cedex, France.

$18{ }^{5}$ University of Science and Technology of Hanoi, Training and Services Building, Vietnam

19 Academy of Science and Technology, 18 Hoang Quoc Viet, Cau Giay District, Hanoi,

20 VietNam

21

$22{ }^{*}$ corresponding authors: Eric Cascales (cascales@imm.cnrs.fr) and Christian Cambillau

23 (cambillau@afmb.univ-mrs.fr). 
24 Running title: T6SS-bacteriophage baseplate homology

25 Keywords: Type VI secretion, nanobody, protein secretion, protein transport, baseplate,

26 structure-function analysis, contractile tail, receptor binding protein, shoulder, tail assembly

27 


\section{ABSTRACT}

The Type VI secretion system (T6SS) is a multiprotein machine widespread in Gram-negative bacteria that delivers toxins into both eukaryotic and prokaryotic cells. The mechanism of action of the T6SS is comparable to that of contractile myophages. The T6SS builds a tail-like structure made of an inner tube wrapped by a sheath assembled under an extended conformation. Contraction of the sheath propels the inner tube toward the target cell. The T6SS tail is assembled on a platform - the baseplate functionally similar to bacteriophage baseplates. In addition, the baseplate docks the tail to a trans-envelope membrane complex that orients the tail toward the target. Here, we report the crystal structure of TssK, a central component of the T6SS baseplate. We show that TssK is constituted of three domains and establish the contribution of each domain to the interaction with TssK partners. Importantly, this study reveals that the $\mathrm{N}$-terminal domain of TssK is structurally homologous to the shoulder domain of phage receptor binding proteins while the $\mathrm{C}$-terminal domain binds the membrane complex. We propose that TssK has conserved the domain of attachment to the virion particle but has evolved the reception domain to use the T6SS membrane complex as receptor. 


\section{Introduction}

Delivery of bacterial effector proteins and toxins into target cells relies on transenvelope nanomachines called secretion systems. These machines select and transport effectors in the milieu or directly into the target cell ${ }^{1}$. Most of these secretion systems evolved from efflux pumps or from machineries involved in conjugation or flagellar, twitching or gliding motility ${ }^{1}$. The Type VI secretion system (T6SS) is a fascinating machine that uses a contractile mechanism similar to that of contractile tail structures such as bacteriophages or R-pyocins ${ }^{2-7}$. The T6SS delivers toxins and effectors in both eukaryotic and procaryotic cells and participates to bacterial pathogenesis and inter-bacterial competition ${ }^{8,9}$. By eliminating competing bacteria, the T6SS confers an increased ability to colonize the niche $\mathrm{e}^{10-16}$

Basically, the T6SS can be viewed as a contractile tail oriented towards the target cell and anchored to the cell envelope by a membrane complex $(\mathrm{MC})^{3,17}$. The MC is evolutionarily related to a sub-complex associated with Type IVb secretion system ${ }^{18,19}$. The MC is a 1.7MDa trans-envelope structure constituted of three conserved subunits: the TssJ outer membrane lipoprotein and the TssL and TssM inner membrane proteins ${ }^{20-26}$. In several cases, the $\mathrm{MC}$ is properly inserted and anchored to the cell wall by additional proteins with peptidoglycan hydrolysis and peptidoglycan binding properties ${ }^{21,27-29}$. The contractile tail is composed of the inner tube made of hexamers of the Hcp protein, stack on each other ${ }^{30,31}$, tipped by VgrG and surrounded by the contractile sheath made of the TssBC proteins ${ }^{30-32}$. The polymerization of the tail tube/sheath tubular structure is initiated on an assembly platform, the baseplate (BP), the less characterized T6SS sub-complex, and is coordinated by the TssA $\operatorname{protein}^{33-35}$. The T6SS contractile tail shares functional and structural homologies with the tail of several bacteriophages ${ }^{5,6,30,36,37}$. Once the T6SS tail is assembled, the sheath contracts and propels the inner tube/spike needle complex towards the target cell ${ }^{39-42}$, and it has been proposed that this needle complex traverses the cell envelope through the $\mathrm{MC}^{26}$. A recent in 
vivo study identified five components of the BP: TssE, $-\mathrm{F},-\mathrm{G},-\mathrm{K}$ and $\mathrm{VgrG}^{34}$. While TssA

was also identified in this screen, later observations have demonstrated that TssA is not a structural component of the baseplate $\mathrm{per} s \mathrm{e}^{34}$. TssE is a homologue of gp25, a bacteriophage T4 baseplate wedge protein ${ }^{18,19,43}$. By contrast, no tri-dimensional structure is available for TssF, $-\mathrm{G}$ or $-\mathrm{K}$. In silico analyses recently predicted that TssF and TssG share limited homologies with gp6 and gp7 respectively ${ }^{34}$ whereas controversies exist regarding TssK $\mathrm{K}^{44,45}$. Interestingly, T6SS BP sub-complexes could be isolated in Serratia marcescens and uropathogenic Escherichia coli (UPEC) such as the TssKFG or TssKFGE complexes ${ }^{33,45}$. These complexes likely represent the equivalent of wedge complexes of phage baseplates, which assemble around the central gp27-gp5 hub/spike ${ }^{46,47}$. In addition to appear central for the assembly of the T6SS wedges, TssK is a key BP subunit mediating contacts with the cytoplasmic domains of MC components ${ }^{35,48,49}$. Hence, TssK is an essential BP component connecting MC, BP and tail components. However, we still lack structural information on TssK. While we showed that TssK assembles trimeric complexes in enteroaggregative E.coli $(\mathrm{EAEC})^{48}$, a study reported that it assembles trimers and hexamers in S. $_{\text {marcescens }}{ }^{33}$. The available structural information on the EAEC TssK protein (accession number: EC042_4526) consists to the low-resolution $(\sim 26 \AA)$ negative stained electron microscopy structure of the TssK trimer, as well as its SAXS envelope ${ }^{48}$. Despite intensive efforts, neither full-length of cleaved forms of TssK could be crystallized. Here, we report the crystal structure of the fulllength EAEC TssK protein, obtained as a complex with a camelid nanobody that facilitated the crystallisation process. The structure of trimeric TssK reveals an unexpected homology of its N-terminal domain with siphophages RBP shoulders, hence expanding the number of homologous proteins between T6SS and bacteriophages. While TssK N-terminal domain attaches to the rest of T6SS baseplate, the C-terminal domain binds the membrane complex and has evolved to use the T6SS membrane complex as receptor for docking the baseplate. In 
95

96

addition, the flexibility of the TssK C-terminal domain suggests that TssK may establish a flexible link to maintain the anchorage of the baseplate to the T6SS membrane complex before and after tail contraction.

\section{Results}

TssK crystallization is facilitated when complexed to the TssK-specific nb18 nanobody.

Despite extensive efforts, previous attempts to crystallize TssK were unsuccessful. Crystallization of proteins has been previously shown to be facilitated once the protein of interest is complexed to camelid single-chain antibodies, called nanobodies ${ }^{50}$. We sused this approach for the crystallization of the periplasmic domain of the T6SS TssM subunit ${ }^{26,51,52}$. We therefore immunized a llama with purified TssK, and isolated nanobodies that bind TssK: nbK18, nbK25 and nbK27. The crystal structure of nbK18 was determined (Supplementary Figure 1a) and its complexation with TssK was then monitored by Biolayer interferometry (BLI). Kinetic and steady-state analyses defined $\mathrm{K}_{D}$ of $2.4 \mathrm{nM}$ and $3.1 \mathrm{nM}$ respectively.

\section{The TssK structure reveals a three-domain protein organized as a tightly packed trimer.}

The structure of the nbK18-TssK co-crystallized complex was determined at $2.6 \AA$ resolution. The complex contains three TssK molecules to which three nbK18 units are bound (Supplementary Figure 1b). TssK trimerization is in agreement with previous gel filtration data suggesting that TssK forms trimer ${ }^{48}$. Each nanobody interacts with two monomers of TssK and covers $\sim 700 \AA^{2}$ of the accessible surface area of a TssK monomer and $80 \AA^{2}$ of a second monomer. Interaction of nbK 18 with TssK is mainly mediated by nbK18 CDR3 but also by the two other CDRs and the conserved skeleton (Supplementary Figure 1b, 
119 Supplementary Table 2), a feature already observed in other cases ${ }^{53,54}$. NbK18 binds the TssK

120 N-terminal $\beta$-sandwich and more specifically the L4-5 and L6-7 loops (Supplementary

121 Figure 1b inset; Supplementary Table 2).

122 The TssK trimer has the overall structure of an apple core or an hourglass, with two globular 123 domains - the N-terminal shoulder (herefater named $\mathrm{TssK}_{\mathrm{S}}$ ) and C-terminal head $\left(\mathrm{TssK}_{\mathrm{H}}\right)$

124 domains - separated by a helical stalk, the neck $\left(\operatorname{Tss}_{N}\right)$. The TssK trimer is tightly packed, 125 as $1400-1570 \AA^{2}$ of the accessible surface area of each monomer is covered by the two other 126 monomers (Fig. 1a). The structure exhibits high B-factors $\left(\sim 100 \AA^{2}\right)$ and consequently the 127 side-chains of solvent exposed residues are often disordered when not involved in crystal 128 packing contacts. A large segment is missing at the bottom of the shoulder domain, between 129 residues 130 and 144 , and a short loop is incomplete at the top of the neck domain, between 130 residues 221 and 224. These segments and have not been incorporated into the model (Fig.

131 1a). Two of the three monomers are not complete: their amino acid chains start at residue 19 132 and end at residues 320 and 315 for monomers $\mathrm{A}$ and $\mathrm{C}$, respectively, suggesting that their C133 terminal domain might be totally disordered. In contrast, the main-chain of monomer B could 134 be traced up to residue 334, and the side-chain identity can be assigned (Fig. 1a). However, 135 despite its stabilization by crystal contacts, the C-terminal domain of chain B exhibits an 136 average B-factor double compared to the rest of the structure, impeding its complete 137 assignment (Fig. 1a).

138 The TssK monomer structure comprises three domains: a N-terminal $\beta$-sandwich domain 139 (shoulder, TssK , residues 19-174), a linker (residues 175-193) and a four-helix bundle 140 middle domain (neck, $\mathrm{TssK}_{\mathrm{N}}$, residues 194-313), and, in chain B only, a partially traced C141 terminal domain (head, $\mathrm{TssK}_{\mathrm{H}}$, residues 315-447) (Fig. 1b). The N-terminal domain starts 142 with a long $\alpha$-helix followed by three anti-parallel $\beta$-strands forming a $\beta$-sheet ( $\beta 1-\beta 3)$. After $143 \beta$-strand 4 , a short $\beta$-hairpin $(\beta 4, \beta 5)$ is followed by $\beta$-strands 6 and 7 . Together, $\beta$-strands 4,6 
144 and 7 form the second $\beta$-sheet of the $\beta$-sandwich. After a long linker, $\alpha$-helices 2,3 and 4

145 form an antiparallel bundle. Helix 4 is then followed by a long unstructured stretch of

146 residues returning in the direction of the $\mathrm{N}$-terminus and positioning $\alpha$-helix 5 parallel to $\alpha$ -

147 helix 3, allowing the chain to continue in a direction opposite to the N-terminus. The C-

148 terminal domain, starting at residue 317 , is predicted by JPRED $^{55}$ as assembling three $\alpha$ -

149 helices and seven $\beta$-strands. We assigned 106 amino acids for a total of 125 for the complete

150 C-terminal domain. At this stage it comprised two complete $\alpha$-helices and an incomplete one,

151 as well as two $\beta$-sheets forming a $\beta$-sandwich of putatively $7 \beta$-strands, but most connections

152 were not visible in the electron density map.

153 To obtain a better model of TssK, the C-terminal domain (amino-acids 316-445) was

154 produced, crystallized and its structure solved at $1.6 \AA$ resolution. This domain is very

155 compact and comprises three $\alpha$-helices $(\alpha 6-8)$ and $7 \beta$-strands $(\beta 10-16)$ (Fig. 1b). The

156 structure of the isolated C-terminal domain was reintroduced in the structure of full-length

157 TssK, and refinement was performed. Apart from loop 372-382, which has a different

158 conformation, the domain conformations are very close in the isolated and full-length

159 structures (r.m.s.d $=1.2 \AA$ on 115 residues) (Supplementary Figure 2). Notably, the C-

160 terminal domain of chain B establishes stabilizing packing contacts, involving mainly loop

$161372-382$. As such, the complete TssK trimer is $\sim 110 \AA$ long and $\sim 85 \AA$ wide at the level of

162 the shoulder domains, and $\sim 40 \AA$ wide at the level of the central domain (Fig. 1a).

163 We previously reported the negative stain electron microscopy (EM) structure of TssK ${ }^{48}$. The

164 crystal structure of TssK was fitted into the EM map using Chimera ${ }^{56}$ (Fig. 1c,d). With the

165 exception of the C-terminal domain of monomer B, TssK fits exquisitely well into the EM

166 structure with a correlation of 0.89 and $93 \%$ of atoms included in the map.

167 Nanobody epitope analysis performed by BLI revealed that nbK27 and nbK25 bind

168 TssK using another epitope compared to nbK18. We therefore co-crystallized the cloned 
169 TssK $_{\mathrm{SN}}$ domain (shoulders and neck domain, residues 1-315) with nbK18 and nbK27. Crystals

170 of the TssK $\mathrm{SN}_{\mathrm{S}}-\mathrm{nbK} 18-\mathrm{nbK} 27$ were obtained and the structure was solved (Supplementary

171 Table 1). The structure of the $\mathrm{TssK}_{\mathrm{SN}}-\mathrm{nbK} 18-\mathrm{nbK} 27$ superimposes well with that of the

172 TssK-nbK18 complex, with a small rotation of the three nbK18. By contrats to nbK18, that

173 binds each TssK monomer, a unique nbK27 binds the trimeric assembly along the 3-fold axis

174 at the bottom of the shoulder domains (Fig. 2a,b), resulting in a TssK $3 / n b K 18_{3} / \mathrm{nbK}_{2} 7_{1}$

175 stoichiometry. The most striking result of nbK27 binding to TssK is the ordering of the

176 bottom of the shoulder domains segments that were disordered in the TssK-nbK18 complex:

177 the N-terminal segment 1-19 and the $\beta$-hairpin 130-144 (Fig. 2c,d). This ordering results in

178 strong domain-swapped like interactions of the 1-19 segment with the i-1 monomer, and of

179 the 130-144 $\beta$-hairpin with the $\mathrm{i}+1$ monomer (Fig. 2c). Notably, the stabilized structures

180 comprise all the bottom part of TssK, a surface with a diameter of $\sim 50 \AA$ (Fig. 2 d). Finally,

181 the list of domains or segments present in the different crystal structures is summarized as a

182 linear sequence representation with secondary structures (Supplementary Figure 3).

The TssK N-terminal domain shares structure similarities with phage Receptor Binding

Protein shoulder domains.

The three domains of TssK were subjected to DALI analyses to detect structural

187 similarities. Interestingly, DALI retrieved strong similarities between the TssK N-terminal $\beta$ sandwich domain and the shoulder domains from lactococcal siphophages receptor binding proteins (RBP) (Fig. 3), including phages 1358 (PDB: 4L9B, Z=9.0, r.m.s.d. $=3.0 \AA{ }^{57}$ ) and p2 (PDB: 1ZRU, Z=6.0 r.m.s.d.=3.0 ; $^{58}$ ). In addition, although helix bundles represent a common structural fold, DALI analyses detected similarities between the TssK neck domain

192 and a domain of the human adenylosuccinate lyase (PDB: 4FFX, Z=9.4, r.m.s.d.=3.1 $\AA{ }^{59}$ )

193 (Supplementary Figure 4). 
Previous studies have shown that TssK is a central subunit of the T6SS, as it interacts with components of the MC (the cytoplasmic domains of TssL $\left(\operatorname{TssL}_{C}\right)$ and TssM $\left(\operatorname{TssM}_{C}\right)$ ), 198 of the baseplate (the TssFG complex) and of the tail (TssA, TssC and Hcp) ${ }^{33,34,39,48,60}$. We therefore sought to define the domains mediating TssK interactions with MC and BP. First, the $\mathrm{N}$-terminal shoulder and neck $\left(\mathrm{TssK}_{\mathrm{SN}}\right)$, shoulder $\left(\mathrm{TssK}_{\mathrm{S}}\right)$, neck $\left(\mathrm{TssK}_{\mathrm{N}}\right)$ and $\mathrm{C}$-terminal

201 head $\left(\mathrm{TssK}_{\mathrm{H}}\right)$ domains of TssK were cloned in two-hybrid vectors and tested for their ability

202 to promote oligomerization and to interact with TssFG, $\operatorname{TssL}_{\mathrm{C}}$ and $\mathrm{TssM}_{\mathrm{C}}(\mathrm{Fig}$. 4a). In

203 agreement with the crystal structure of the TssK trimer (Fig. 1a), both shoulder and neck 204 domains oligomerize whereas the TssK head domain does not interact with TssK (Fig. 4a, 205 first column). More interestingly, the two-hybrid analyses revealed that the $\mathrm{N}$-terminal 206 shoulder domain mediates contacts with the TssFG complex whereas the C-terminal head 207 domain interacts with $\mathrm{TssL}_{\mathrm{C}}, \mathrm{TssM}_{\mathrm{C}}$ and to a lesser extent to TssFG. We then monitored co208 immunoprecipitation experiments. Soluble lysates of cells producing the VSV-G-tagged TssK 209 domains were combined with lysates containing FLAG-tagged TssF and TssG, or the 210 cytoplasmic domains of TssL $\left(\mathrm{TssL}_{\mathrm{C}}\right)$ or TssM $\left(\mathrm{TssM}_{\mathrm{C}}\right)$. FLAG-tagged proteins were 211 immobilized on agarose beads coupled to the monoclonal anti-FLAG antibody and the eluted 212 material was analysed by SDS-PAGE and immuno-staining. Figure $4 \mathrm{~b}$ shows that the $\mathrm{TssK}_{\mathrm{S}}$ 213 domain is co-precipitated with the TssFG complex, whereas the the $\mathrm{TssK}_{\mathrm{H}}$ domain co214 precipitates with $\mathrm{TssL}_{\mathrm{C}}$ and $\mathrm{TssM}_{\mathrm{C}}$ - and to a lower extent - with TssFG. The results of 215 BACTH and co-immunoprecipitation analyses are summarized in Supplementary Figure 5.

216 To confirm these results, we engineered vectors producing TssFG as well as either $2176 \times$ His-tagged $\mathrm{TssK}_{\mathrm{SN}}$ or $\mathrm{TssK}_{\mathrm{H}}$. HPLC chromatography indicated that TssKFG and $218 \mathrm{TssK}_{\mathrm{SN}} \mathrm{FG}$ complexes form (Supplementary Figure 6a). However, while we succeeded to 
purify the $\mathrm{TssK}_{\mathrm{SN}} \mathrm{FG}$ complex (Fig. 4c, Supplementary Figure 6a), TssK $\mathrm{H}_{\mathrm{H}}$ was purified alone

220 and did not co-purify with TssFG; isolated TssFG is not observed on the chromatogram as it

221 is insoluble (Supplementary Figure 7a). These results confirm the $\mathrm{TssK}_{\mathrm{SN}} / \mathrm{TssFG}$ complex

222 interaction and indicate that the low $\mathrm{TssK}_{\mathrm{H}} / \mathrm{TssFG}$ interaction, observed by BACTH and co-

223 immuno-precipitation, should be weaker and in fast exchange. To gain further insights on the

224 contribution of the $\mathrm{TssK}_{\mathrm{SN}}$ domain for binding to the TssFG complex, we then tested binding

225 of the TssK-specific nbK18 and nbK27 nanobodies to the $\mathrm{TssK}_{\mathrm{SN}} \mathrm{FG}$ complex. The purified

$226 \mathrm{TssK}_{\mathrm{SN}} \mathrm{FG}$ complex was mixed with an excess of nanobody and analysed by gel filtration and

227 HPLC. First, we confirmed that nbK18 and nbK27 bind alone and simultaneously to TssK $\mathrm{SN}_{\text {. }}$

228 We performed then the analysis of $\mathrm{TssK}_{\mathrm{SN}} \mathrm{FG}$ in the presence of nbK18, nbK27 and nbK18+

229 nbK27 (Supplementary Figure $6 b$ ). We found that nbK18 binds to the TssK ${ }_{\mathrm{SN}} \mathrm{FG}$ complex

230 (Fig. 4c,d and Supplementary Figure 6c) whereas nbK27 does not (Fig. 4c,e). From these

231 results, we concluded that nbK27 and TssFG share the same binding site to TssK, and hence,

232 that TssFG should bind to the bottom of the $\mathrm{TssK}_{\mathrm{S}}$ domain comprising the residues 1-19

233 segment and the 130-144 $\beta$-hairpin. Taken together, the results of the co-purification and the

234 nanobody binding experiments confirmed the interaction of $\mathrm{TssK}_{\mathrm{S}}$ with the TssFG complex

235 and suggested that TssFG binds the bottom of $\mathrm{TssK}_{\mathrm{S}}$.

236 We could not assay the $\mathrm{TssK}_{\mathrm{H}}$ interaction with the TssMc domain, as it is insoluble. In

237 contrast we performed a gel filtration of a mixture of TssLc and $\mathrm{TssK}_{\mathrm{H}}$, and found that they

238 do not co-purify. As an interaction is observed by double-hybrid and co-immunoprecipitation

239 this latter result suggests that this interaction is rather weak (around 1-10microM) and in fast

240 exchange. Indeed, this does not reflect the biological situation in which the interaction of

241 several TssK trimers and several Lc dimers occurs, leading certainly to a tremendous avidity

242 increase. 


\section{Discussion}

In this work, we present the crystal structure and a domain analyses of a T6SS core component, TssK. Previous studies have demonstrated that TssK is a trimer and is an essential subunit of the Type VI secretion apparatus by connecting the trans-envelope membrane complex to the phage-like contractile tail ${ }^{48}$. In addition, TssK has been shown to be part of the T6SS assembly platform or baseplate ${ }^{33,34,39,45,48}$. It has been recently reported that phage T4 is a very complex phage, and that phage Mu is a simpler, widespread, and a better paradigm of phages with contractile tails ${ }^{61}$. Notably, TssK has no counterpart in phage $\mathrm{Mu}$ whereas other T6SS baseplate components have counterparts in both phages $\mathrm{T} 4{ }^{45}$ and $\mathrm{Mu}^{61}$ : TssE corresponds to gp25 (T4) and Mup46 (Mu), TssF to gp6 and Mup47 and TssG to Mup48 (no homologue in T4).

The X-ray structure of TssK confirms that TssK exists as a trimer. The TssK trimer has an overall architecture resembling that of an apple core: a shoulder base and a distal head separated by the central neck. The structure of TssK is modular. It comprises three domains: a $\mathrm{N}$-terminal domain, essentially $\beta$-stranded, comparable to the $\mathrm{N}$-terminal shoulder domains of phages $\mathrm{p} 2$ and 1358 RBPs, followed by a $\alpha$-helical central domain and a mixed $\alpha \quad \beta C$ terminal globular domain. In vivo interactions studies with known partners of TssK support the idea that TssK acts as a connector between two T6SS complexes that are evolutionarily unrelated: the phage-like baseplate/tail and the T4bSS IcmF/DotU-like membrane complex (Fig. 5). From the protein-protein interaction assays we concluded that the TssK N-terminal shoulder domain binds to TssFG and that the $\mathrm{C}$-terminal head domain mediates interactions with the TssL and TssM inner membrane proteins..

The fascinating observation of this study is that TssK shares a similar overall architecture with lactococcal siphophage receptor-binding proteins. These RBPs are trimers and comprise shoulder and head domains separated by the neck ${ }^{57,58,62}$. They recognize the 
269 host cell surface and are responsible for properly orienting the phage onto the host cell ${ }^{63,64}$.

270 RBPs are anchored to the virion and bind to receptors at the cell surface of the target cell.

271 Anchorage to the virion particle is mediated by the shoulder domain whereas the ability to

272 recognize the cell envelope receptor is conferred by the head domain. It has been reported that

273 in each lactococcal phage family, the shoulder domain structure is strictly conserved whereas

274 the head domain is relatively diverse and confers specificity for binding to the proper target

275 cell $^{57,58,62,63,65}$. Another striking similarity between the TssK and RBP shoulder domains is the

276 ordering of their bottom segments upon binding to partners. When the phage $\mathrm{p} 2 \mathrm{RBP}$ is

277 expressed alone, the $20 \mathrm{~N}$-terminal residues are disordered and not visible in the electron map

278 density. In contrast, they are ordered when the RBP is complexed with the other baseplate

279 components and constitutes the main contributors to the interaction. Worth noticing, the "arm

280 and hand" domain of the Dit protein inserts between the three N-termini, holding thus the

281 RBP trimer ${ }^{58,62,65}$. A similar situation may exist with TssK. Although we do not have the

282 structure of TssK within the TssKFG complex, nanobody binding experiments on the

283 TssKFG complex have shown that the TssFG binding site on TssK involves the bottom of the

284 trimer, including the N-termini (Supplementary Figure 8). The ordering of this site upon

285 nbK27 binding suggests that this nanobody acts as a surrogate of the TssFG complex.

286 In myophages, such as phages $\mathrm{T} 4$ or $\mathrm{Mu}$, both the baseplate and the tail sheath change

287 conformation upon contraction ${ }^{45,61}$. The tail sheath conformational change is also

288 documented for the $\mathrm{T} \mathrm{SS}^{32}$. It is therefore expected that a conformational reorganization also

289 occurs for the T6SS baseplate ${ }^{35,38}$. The flexibility of TssK head domain relative to the rest of

290 the trimer suggests that TssK may constitute a flexible link between the T6SS baseplate and

291 its membrane domain to accommodate the different conformations and radii before and after

292 tail contraction. The TssK flexible hinge would therefore prevent disruption of the contact

293 between the membrane and the baseplate complexes during sheath contraction. 
294 By sharing the overall architecture and the N-terminal shoulder domain with RBPs, we

295 propose that TssK represents a RBP-like component of T6SS. Such as in RBPs, the TssK has

296 a modular architecture with a N-terminal shoulder domain and a C-terminal head domain.

297 The TssK shoulder domain interacts with the other T6SS baseplate components (TssFG). By

298 contrast, the head domain of TssK mediates interaction with the cytoplasmic domains of TssL

299 and TssM and hence docks the baseplate to the MC (Fig. 5). These data are consistent with

300 previous fluorescence microscopy imaging demonstrating that GFP-labeled TssK is recruited

301 to the MC and is necessary for recruiting the gp6/Mup47-like TssF baseplate subunit ${ }^{34}$. Based

302 on these data and on the observation of TssE and TssG interactions with the cytoplasmic

303 domains of TssL and TssM respectively ${ }^{34,49,66}$, we propose that TssK first interacts with the

$304 \mathrm{MC}$ and that TssE-TssL and TssG-TssL additional contacts stabilize the MC-BP complex.

305 TssK has therefore a similar function to RBPs: it is anchored to the phage-like structure by a

306 conserved shoulder domain but, instead of allowing the phage particle to bind to the target

307 cell surface, it has evolved a distinct head domain to recognize and bind to the T6SS MC. It

308 should be stressed that while TssFG have sequence similarity with Myoviridae phages

309 baseplate components, the TssK shoulder domain has structural similarity with Siphoviridae

310 phages RBPs, and has no counterpart in Myoviridae. Therefore, even if the origin of T6SS tail

311 and baseplate is from Myoviridae phages, the TssK N-terminal domain may derive from

312 horizontal transfer from a Siphoviridae component in order to provide the ability to bind the

313 TssJLM complex of T6SS). These results point to a more complicated evolutionary process

314 leading to the T6SS where elements of different phage types were combined.

315

316 Materials and Methods

317

318 Bacterial strains, growth conditions and chemicals 
319 The strains used in this study are listed in Supplementary Table 3. Escherichia coli K-12

320 strains DH5 $\alpha$, W3110, BTH101 and T7 Iq pLys were used for cloning procedures, co-

321 immune-precipitation, bacterial two-hybrid and protein purification respectively. The

322 enteroaggregative E. coli (EAEC) wild-type O3:H2 17-2 strain genomic DNA was used as

323 template for cloning. E. coli $\mathrm{K}-12$ and EAEC cells were routinely grown in LB broth at $37^{\circ} \mathrm{C}$,

324 with aeration. For protein production, cells were grown in terrific broth. Plasmids and

325 mutations were maintained by the addition of ampicillin $(100 \mu \mathrm{g} / \mathrm{mL})$, kanamycin $(50 \mu \mathrm{g} / \mathrm{mL})$

326 or chloramphenicol $(40 \mu \mathrm{g} / \mathrm{mL})$. Gene expression was induced by the addition of iso-propyl-

$327 \beta$-D-thio-galactopyranoside (IPTG, Sigma-Aldrich, $1 \mathrm{mM}$ for nanobodies, $0.5 \mathrm{mM}$ for TssK

328 and TssK domains production and BACTH analyses), L-arabinose (Sigma-Aldrich; 0.2\%) or

329 anhydrotetracyclin (AHT; IBA Technologies; $0.2 \mu \mathrm{g} / \mathrm{mL}$ ).

\section{Plasmid construction}

332 PCR were performed with a Biometra thermocycler, using the Pfu Turbo DNA polymerase

333 (Stratagene; La Jolla, CA). Plasmids and oligonucleotides are listed in Supplementary Table

334 3. Plasmids producing EAEC TssK domains fused to a VSV-G tag or to the T18 or T25

335 domains of the $B$. pertussis adenylate cyclase were engineered by restriction-free cloning ${ }^{67}$.

336 Briefly, DNA fragments corresponding to TssK domains were amplified from EAEC 17-2

337 genomic DNA using oligonucleotides bearing 5' extensions annealing on the target plasmid.

338 The PCR products were then used as primers for amplification of the target plasmid.

339 Constructs have been screened by colony-PCR and verified by DNA sequencing. The TssK $\mathrm{H}_{\mathrm{H}}$

340 domain (residues S316 to T445) was cloned into the pETG20A expression vector using the 341 same procedure. 
342 The $t s s K, t s s F$ and $t s s G$ were initially cloned in a pCDF-Duetl vector with Cter His ${ }_{6}{ }_{\text {tag, }}$ Nter

343 TREP tag and Cter FLAG tag for The $t s s K$, $t s s F$ and $t s s G$, respectively (Supplementary

344 Figure 8). For this study, the TREP and FLAG tags were removed by overlapping PCR

345 (Supplementary Figure 8). The $t s s K_{S N} F G$ and $t s s K_{H} F G$ vectors were obtained by Gibson

346 assembly (Gibson Assembly Cloning Kit, New England BioLabs) (Supplementary Figure 9).

Bacterial two-hybrid

349 The adenylate cyclase-based bacterial two-hybrid technique ${ }^{68}$ was used as previously

350 published ${ }^{69}$. Briefly, the proteins to be tested were fused to the isolated T18 and T25

351 catalytic domains of the Bordetella adenylate cyclase. After introduction of the two plasmids

352 producing the fusion proteins into the reporter BTH101 strain, plates were incubated at $30^{\circ} \mathrm{C}$

353 for 24 hours. Three independent colonies for each transformation were inoculated into $600 \mu \mathrm{L}$

354 of LB medium supplemented with ampicillin, kanamycin and IPTG (0.5 mM). After

355 overnight growth at $30^{\circ} \mathrm{C}, 10 \mu \mathrm{L}$ of each culture were dropped onto LB plates supplemented

356 with ampicillin, kanamycin, IPTG and 5-bromo-4-chloro-3-indolyl- $\beta$-D-galactopyranoside

357 (X-Gal) and incubated for 12 hours at $30{ }^{\circ} \mathrm{C}$. Controls include interaction assays with TolB

358 and Pal, two protein partners unrelated to the T6SS. The experiments were done at least in

359 triplicate and a representative result is shown.

\section{Co-immunoprecipitation}

361 Co-immunoprecipitation experiments were performed as previously described ${ }^{35} \cdot 10^{11}$

362 exponentially growing cells producing the proteins of interest were harvested, and

363 resuspended in CellLytic ${ }^{\mathrm{TM}}$ B Cell Lysis reagent (Sigma-Aldrich) supplemented with protease

364 inhibitors (Complete, Roche), lysozyme $(100 \mu \mathrm{g} / \mathrm{mL})$ and DNase $(100 \mu \mathrm{g} / \mathrm{mL})$ and incubated 
365 for 1 hour with strong shaking. The insoluble material was discarded by centrifugation for 45

366 min at $60,000 \times g$ and the supernatant from $2 \times 10^{10}$ cells was incubated overnight at $4{ }^{\circ} \mathrm{C}$ with

367 anti-FLAG M2 affinity beads (Sigma-Aldrich). Beads were then washed twice with

368 CellLytic $^{\mathrm{TM}}$ and once with Tris- $\mathrm{HCl} 20 \mathrm{mM}$ pH8.0, $\mathrm{NaCl} 100 \mathrm{mM}$. The total extract and

369 immunoprecipitated material were resuspended and boiled in Laemmli loading buffer prior to

370 analyses by SDS-PAGE and immunoblotting.

371 High-performance liquid size-exclusion chromatography

372

373 Size exclusion chromatography was performed on an Ultimate 3000 HPLC system (Dionex),

374 using a Superdex 200 increase 10/30GL column equilibrated in Tris $10 \mathrm{mM}$ pH8, NaCl 150

$375 \mathrm{mM}$ at a flow rate of $0.6 \mathrm{ml} / \mathrm{min}$. Accurate injections of $30 \mathrm{ul}$ samples were done using the

376 autosampler and detection was monitored at $280 \mathrm{~nm}$.

377

378 Gel filtration analyses

379 The effect of domains deletions on TssKFG as well as the binding of nanobodies nbK18 and

380 nbK27 to $\mathrm{TssK}_{\mathrm{SN}} \mathrm{FG}$ were analyzed using nickel affinity chromatography and gel filtration

381 chromatography. The three constructs possess a unique module bearing a $\mathrm{His}_{6}$ tag

382 (Supplementary Figure 9)): On TssK for the TssKFG construct, on $\mathrm{TssK}_{\mathrm{H}}$ for the TssK $\mathrm{H}_{\mathrm{H}} \mathrm{FG}$

383 construct, and on $\mathrm{TssK}_{\mathrm{SN}}$ for the $\mathrm{TssK}_{\mathrm{NS}} \mathrm{FG}$ construct. The purification procedures were

384 similar to those used for the TssK-nbK18 complex (see above). An molar excess of nanobody

385 (1.2), nbK18 or nbK25, was added to the TssK ${ }_{\mathrm{SN}} \mathrm{FG}$ complex and submitted to a nickel

386 affinity purification, followed by gel filtration. The mixtures were immobilized on a 5-mL

$387 \mathrm{Ni}^{2+}$ HisTrap prepacked column (GE Healthcare) using an AKTA FPLC system. After

388 extensive washing steps in absence or presence of $50 \mathrm{mM}$ of imidazole, they were eluted with 
$250 \mathrm{mM}$ imidazole. The fractions containing the $\mathrm{His}_{6}$ tagged proteins were pooled and loaded

390 on to a preparative 10/300 Superdex 200 gel filtration column (GE Healthcare) equilibrated in

$39120 \mathrm{mM}$ Tris- $\mathrm{HCl}, \mathrm{pH} 8.0,150 \mathrm{mM} \mathrm{NaCl}$. The peaks were analysed by SDS gels. The same

392 conditions were applied to the $\mathrm{Tss}_{\mathrm{H}} \mathrm{FG}$ construct. The fractions containing the $\mathrm{His}_{6}$ tagged

393 proteins were pooled and loaded on to aHiLoad 16/600 Superdex 200, column equilibrated in

$39420 \mathrm{mM}$ Tris- $\mathrm{HCl} \mathrm{pH}$ 8.0, $150 \mathrm{mM} \mathrm{NaCl}$ buffer.

\section{Protein purification.}

$396 \quad 6 \times$ His-tagged TssK was purified as previously described ${ }^{48}$. E. coli T7 Iq pLys cells carrying

397 pRSF-TssK (TssK 6 His $)$ or pETG20A-TssK ${ }_{\mathrm{Ct}}$ (TssK C-terminal domain fused to a thioredoxin-

$3986 \times$ His-TEV N-terminal extension, TRX-6His-TEV-TssK $\mathrm{C}_{\mathrm{Ct}}$ ) were grown at $37^{\circ} \mathrm{C}$ in terrific

399 broth to an optimal optical density $\left(\mathrm{OD}_{600}\right) \sim 0.6-1.0$ and $t s s K$ expression was induced with

$400 \quad 0.5 \mathrm{mM}$ IPTG for $16 \mathrm{~h}$ at $17^{\circ} \mathrm{C}$. Cells were harvested, resuspended in $50 \mathrm{mM}$ Tris- $\mathrm{HCl} \mathrm{pH}$

$4018.0,300 \mathrm{mM} \mathrm{NaCl}$ supplemented with $1 \mathrm{mM}$ PMSF, $0.25 \mathrm{mg} / \mathrm{mL}$ lysozyme, $100 \mu \mathrm{g} / \mathrm{mL}$

402 DNase I and $20 \mathrm{mM} \mathrm{MgCl}_{2}$. After sonication, soluble proteins were separated from inclusion

403 bodies and cell debris by centrifugation at $20,000 \times g$ for $30 \mathrm{~min}$. TssK $6 \mathrm{His}$ and TRX-6His-

404 TEV-TssK $\mathrm{Ct}_{\mathrm{t}}$ were immobilized on a $5-\mathrm{mL} \mathrm{Ni}^{2+}$ His Trap prepacked column (GE Healthcare)

405 using an AKTA FPLC system. After extensive washing steps in absence or presence of 50

$406 \mathrm{mM}$ of imidazole, $\mathrm{TssK}_{6 \mathrm{His}}$ and TRX-6His-TEV-TssK $\mathrm{Ct}_{\mathrm{t}}$ were eluted with $250 \mathrm{mM}$ imidazole.

407 The fractions containing the $\mathrm{TssK}_{6 \mathrm{His}}$ protein were pooled and loaded on to a preparative

408 Superdex 200 gel filtration column (GE Healthcare) equilibrated in $20 \mathrm{mM}$ Tris-HCl, $\mathrm{pH} 8.0$,

$409150 \mathrm{mM} \mathrm{NaCl}$. TssK 6 His was concentrated to $25 \mathrm{mg} / \mathrm{mL}$ and stored at $4{ }^{\circ} \mathrm{C}$ for crystallization

410 trials. The fractions containing TRX-6His-TEV-TssK $\mathrm{Ct}_{\mathrm{t}}$ were pooled and incubated with TEV

411 protease (protein/TEV to a 20/1 molecular ratio) coupled to dialysis against $50 \mathrm{mM}$ Tris- $\mathrm{HCl}$

$412 \mathrm{pH} 8.0,300 \mathrm{mM} \mathrm{NaCl}$ supplemented with $10 \mathrm{mM}$ imidazole overnight at $4{ }^{\circ} \mathrm{C}$. The proteins

413 were then subjected to a second $\mathrm{Ni}^{2+}$ HisTrap prepacked column. The untagged $\mathrm{TssK}_{\mathrm{H}}$ protein 
414 was collected in the flow through and concentrated on a preparative Superdex 200 gel

415 filtration column equilibrated in $20 \mathrm{mM}$ Tris- $\mathrm{HCl}, \mathrm{pH} 8.0,150 \mathrm{mM} \mathrm{NaCl}$. TssK $\mathrm{H}_{\mathrm{H}}$ was

416 concentrated to $26 \mathrm{mg} / \mathrm{mL}$ and stored at $4{ }^{\circ} \mathrm{C}$ for crystallization trials. pETG20A-TssK $\mathrm{SN}$

417 (TssK N-terminal domain fused to a thioredoxin-6×His-TEV N-terminal extension, TRX-

418 6His-TEV-TssK $\mathrm{SN}_{\mathrm{SN}}$ ) was prepared and purified in the same conditions as $\mathrm{TssK}_{6 \mathrm{His}}$.

Generation and purification of TssK-specific llama nanobodies.

To obtain nanobodies against TssK, a llama (Lama glama) was immunized with

422 purified $\mathrm{TssK}_{6 \mathrm{His}}$ (Ardèche-lamas France). Approximately $400 \mu \mathrm{g}$ of $\mathrm{TssK}_{6 \mathrm{His}}$ in $10 \mathrm{mM}$

423 HEPES, $150 \mathrm{mM} \mathrm{NaCl}, \mathrm{pH}$ 7.5, was injected subcutaneously four times at one-week intervals

424 using incomplete Freund's adjuvant, followed by a fifth injection two weeks later. Blood

425 samples were collected aseptically 5 days after the last boost. Lymphocytes were isolated

426 from blood samples, and cDNA was synthesized from the acquired RNA using a reverse PCR

427 protocol. A nanobody phage display library of approximately $10^{9}$ independent transformants

428 was generated using the phagemid vector $\mathrm{pHEN} 4^{70,71}$. Phage display selection and screening

429 of specific nanobodies were performed as previously published ${ }^{72}$. An enrichment of antigen-

430 specific clones was observed after two consecutive rounds of selection on solid-phase coated

431 antigen. After the second round, TssK-specific nanobodies were identified, and the inserts of

432 the corresponding pHEN4-derived plasmids were sequenced and cloned into the pHEN6

433 vector. E. coli WK6 cells carrying the pHEN6 derivatives were grown at $37^{\circ} \mathrm{C}$ in terrific broth

434 supplemented with $0.1 \%$ glucose and $100 \mu \mathrm{g} / \mathrm{mL}$ ampicillin to an optical density $\sim 0.6-1.0$

435 and the expression of the nanobodies was induced by the addition of $1 \mathrm{mM}$ IPTG for $16 \mathrm{~h}$ at

$43628^{\circ} \mathrm{C}$. The periplasmic fraction containing the nanobodies was prepared using mild osmotic

437 shock and the His-tagged nanobodies were immobilized on a 5-mL Ni-NTA column

438 equilibrated in $50 \mathrm{mM}$ Tris- $\mathrm{HCl}$, $\mathrm{pH} 8.0,300 \mathrm{mM} \mathrm{NaCl}$, and $10 \mathrm{mM}$ imidazole. Nanobodies 
were eluted in $250 \mathrm{mM}$ imidazole and concentrated using the Amicon-technology $(10-\mathrm{kDa}$

440 cut-off) prior to loading on a HiLoad 16/60 Superdex 75 gel filtration column equilibrated in $20 \mathrm{mM}$ Tris- $\mathrm{HCl}, \mathrm{pH} 8.0,150 \mathrm{mM} \mathrm{NaCl}$. The final concentration for NbK18 and $\mathrm{NbK} 27$ were $13 \mathrm{mg} / \mathrm{mL}$ and $10 \mathrm{mg} / \mathrm{mL}$, respectively.

Crystallization screening experiments were performed with several commercial kits, including STURA, WIZARD and MDL. The nanodrop crystallization experiments were performed in SWISSCI 3-well plates. The reservoirs of the SWISSCI plates were filled up using a TECAN pipetting robot, while the nanodrops were dispensed with a Mosquito robot. All crystallization experiments were performed at $293 \mathrm{~K}$. For nbK18, a single crystal was obtained by mixing $100 \mathrm{~nL}$ of the nbK18 protein solution with $100 \mathrm{~nL}$ of $0.2 \mathrm{M} \mathrm{Li}_{2} \mathrm{SO}_{4}, 0.1$ $\mathrm{M} \mathrm{NaAc}, \mathrm{pH} 4.75$, and $30 \% \mathrm{~m} / \mathrm{v}$ PEG8000. The crystal was cryo-cooled in reservoir liquid supplemented with $5 \%$ ethylene glycol. For the TssK-nbK18 complex, purified TssK was mixed with nbK18 (TssK:nbK18 at 1:1.2 molecular ratio), and then adjusted to a

455 concentration of $10 \mathrm{mg} / \mathrm{mL}$ for 1 hour prior to crystallization experiments. Small crystals were obtained in $100 \mathrm{mM}$ Tris-HCl, $\mathrm{pH} 8.5,200 \mathrm{mM} \mathrm{MgCl}_{2}, 15 \%$ PEG-6000. Optimization was achieved by varying the concentration of PEG- 6000 from 8 to $20 \% \mathrm{~m} / \mathrm{v}$ and the $\mathrm{pH}$ from 8 to 9. Quality crystals were obtained in $100 \mathrm{mM}$ Tris- $\mathrm{HCl}$, $\mathrm{pH} 8.3,200 \mathrm{mM} \mathrm{MgCl}, 10 \%$ PEG-6000. All crystals, including CsI/NaI derivatives, were cryo-cooled in a well solution supplemented with $20 \%$ ethylene glycol. For the CsI/NaI derivatives, the TssK-nb18 crystals

461 were soaked for a few seconds in a well solution supplemented with $20 \%$ ethylene glycol and $4620.5 \mathrm{M} \mathrm{CsI} / \mathrm{NaI}$. For TssK $\mathrm{H}$, the best crystals appeared in $400 \mathrm{mM} \mathrm{NaH}_{2} \mathrm{PO}_{4}, 1.6 \mathrm{M} \mathrm{K}_{2} \mathrm{HPO}_{4}$, $463100 \mathrm{mM}$ imidazole, $\mathrm{pH}$ 8.0. Crystals were flash cooled in $5 \mathrm{M}$ sodium formate. Crystals of the 
464 TssK $_{\mathrm{SN}}-\mathrm{nbK} 18-\mathrm{nbK} 27$ complex were obtained in the STURA Footprint Screening plate.

$465 \operatorname{TssK}_{\mathrm{SN}}(35 \mathrm{mg} / \mathrm{ml})$ was mixed with the purified nanobodies nbK18 and nbK27 concentrations

466 of $10 \mathrm{mg} / \mathrm{ml}$, with a molar excess of $1.2 v s \mathrm{TssK}_{\mathrm{SN}}$. A volume of $100 \mathrm{~nL}$ of the proteins

467 mixtures was mixed with 45\% PEG600, 0.1 M HEPES pH 7.5, and disposed over the

468 reservoir. Crystals appear in a few days. Crystals were cryo-cooled in the mother liquor.

469 Data collection was performed at ID23-2 (ESRF synchrotron, Grenoble, France) for nbK18

470 and $\mathrm{TssK}_{\mathrm{H}}$, and at Proxima 1 (Soleil synchrotron, Saint-Aubin, France) for the TssK-nbK18

471 complex and the data for the crystal of the TssK $\mathrm{SN}_{\mathrm{SN}}-\mathrm{nbK} 18-\mathrm{nbK} 27$ complex were collected at

472 ESRF beam line ID30A-3 (Supplementary Table 1). The data were integrated and scaled

473 with the XDS/XSCALE package and converted to a ccp4 input format file by XDSCONV ${ }^{73}$.

474 For nbK18, the crystal diffracted up to $1.5 \AA$ (Supplementary Table 1 ) and belonged to space

475 group $\mathrm{P}_{3}$, with cell dimensions $\mathrm{a}=\mathrm{b}=53.4 \AA, \mathrm{c}=88.0 \AA ; \alpha=\beta=\gamma=90^{\circ}$. Two molecules

476 in the symmetric unit yielded a Vm value of $2.29 \AA 3 / \mathrm{Da}$ and $46.35 \%$ solvent. Molecular

477 replacement was performed using MOLREP ${ }^{74}$ and a truncated nanobody structure as a

478 starting model. Refinement was performed using autoBUSTER ${ }^{75}$ alternating with rebuilding

479 with COOT $^{76}$. Two nbK18 molecules are contained in an asymmetric unit and possesses a

480 shorter complementary-determining region 3 (CDR3) compared to those usually found in

481 camelid single-chain domains. For TssK-nbK18, the crystal belonged to the orthorhombic

482 space group $\mathrm{P} 2{ }_{1} 2{ }_{1} 2_{1}$ with cell dimensions $\mathrm{a}=93.3, \mathrm{~b}=153.7, \mathrm{~b}=154.8 \AA ; \alpha=\beta=\gamma=90^{\circ}$.

483 Diffraction images were processed and scaled with the XDS/XSCALE package ${ }^{73}$. The high-

484 resolution data cut-offs were defined based on the CC1/2 statistical indicator ${ }^{77}$. Molecular

485 replacement on the native data set using the structure of nbK18 provided the positions of three

486 nbK18, but this did not yield a good enough initial electron density map. Therefore, three

487 360-degree rotation datasets were collected at different positions of the large single CsI/NaI

488 crystal derivative at $1.7712-\AA \AA$ X-ray wavelength to $3.5-\AA$ resolution. The heavy atom 
substructure, comprising 20 sites, was obtained with the SHELXC/D/E software suite ${ }^{78}$ using

490 the HKL2MAP graphical interface ${ }^{79}$. This sub-structure was subsequently refined and used

491 for phase calculation with PHASER $^{80}$. Phase improvement and extension by density

492 modification using 3-fold non-crystallographic symmetry averaging performed with

493 PARROT $^{81}$ produced readily interpretable maps that allowed positioning of the three nbK18

494 and a first rough TssK model to be built. Inspection of electron density maps and model

495 adjustment and rebuilding were performed using $\mathrm{COOT}^{76}$, and BUSTER was used for model

496 refinements ${ }^{75}$. The initial refined model was then positioned by molecular replacement with

497 MOLREP $^{74}$ in a $2.7 \AA$ resolution native dataset collected at 0.9786 - $\AA$ wavelength with cell

498 dimensions $\mathrm{a}=93.3, \mathrm{~b}=153.7, \mathrm{~b}=154.8 \AA$. Then, several iterations of model improvement were

499 conducted by cycling through refinement with autoBUSTER ${ }^{75}$, phase improvement by density

500 modification with PARROT ${ }^{81}$, auto-tracing with BUCCANEER ${ }^{82}$ and manual pruning and

501 rebuilding using $\mathrm{COOT}^{76}$. The structure of $\mathrm{TssK}_{\mathrm{H}}$ was obtained by molecular replacement

502 using MOLREP ${ }^{74}$ using the partial structure in the refined full-length TssK and a 1.6- $\AA$

503 resolution dataset. Refinement and manual building were performed as described above. The

$504 \mathrm{TssK}_{\mathrm{SN}}-\mathrm{nbK} 18-\mathrm{nbK} 27$ data were processed and scaled with the XDS/XSCALE package ${ }^{73}$.

505 The crystald belong to the same space group as TssK-nbK18 (P2 $\left.2_{1} 2_{1}\right)$ with a comparable

506 packing and cell dimensions of $\mathrm{a}=90.9, \mathrm{~b}=143.3, \mathrm{c}=150.3$. The structure was determined by

507 molecular replacement with MOLREP $^{74}$ using the TssK $_{\mathrm{SN}}$ and nbK18 structures as separate

508 models. The resulting map made it possible to complete the missing segments of $\mathrm{TssK}_{\mathrm{SN}}(1-$

50919 and 130-144) as well to construct the nbK27 model using alternate manual fitting with

$510 \mathrm{COOT}^{76}$ and autoBUSTER ${ }^{75}$ for model refinements at $2.2 \AA$ resolution (Supplementary Table 511 1). 
NbK18 was biotinylated using the EZ-Link NHS-PEG4-Biotin kit (Perbio Science,

515 France). The reaction was stopped by removing the excess biotin reagent using a Zeba Spin

516 Desalting column (Perbio Science, France). BLI assays were performed in black 96-well

517 plates using an OctetRed96 (ForteBio, USA) apparatus. The total working volume for

518 samples or buffer was $0.2 \mathrm{ml}$ and the rpm setting was $1000 \mathrm{rpm}$ for baseline, loading, and

519 association and dissociation steps. The experiments were performed at $25^{\circ} \mathrm{C}$. Prior to each

520 assay, streptavidin (SA) biosensor tips (ForteBio, USA) were hydrated in $0.2 \mathrm{ml}$ kinetic

521 buffer (KB, ForteBio, USA) for 20 min. Streptavidin (SA) biosensor tips were then loaded

522 with biotinylated $\mathrm{nbK} 18$ at $5 \mathrm{mg} / \mathrm{mL}$ in $\mathrm{KB}$, followed by a quenching step using biocytin. A

523 baseline was recorded, and nbK18 binding to $\mathrm{TssK}_{6 \mathrm{His}}$ was performed at concentrations of

5240.08 to $50 \mathrm{nM}$. Association and dissociation were carried out for $300 \mathrm{~s}$ and $600 \mathrm{~s}$,

525 respectively. Complete dissociation of the complex was achieved by three-fold regeneration

526 (5 s in glycine $10 \mathrm{mM}, \mathrm{pH} 1.7)$ and neutralization $(5 \mathrm{~s}$ in $\mathrm{KB})$.

\section{Data availability and accession codes}

529 Structures of nbK18, TssK-nbK18 complex, TssK ${ }_{\mathrm{SN}}-\mathrm{nbK} 18-\mathrm{nbK} 27$ complex and $\mathrm{TssK}_{\mathrm{H}}$ were

530 deposited in the Protein Data Bank (PDB) under accession numbers 5M2W, 5M30, 5MWN

531 and 5M2Y, respectively.

\section{Author contributions}

534 V.S.N., E.C. and C.C. designed the study. V.S.N., L.L., S.S., P.L., T.T.H.P., T.T.N.T., Y.C., 535 A.Z., A.D., E.D., A.R., C.K., E.C. and C.C. contributed to analysis of the data and 536 preparation of this manuscript. V.S.N., S.S., P.L. and C.C. perform the proteins production, 537 crystallization and crystallographic experiments. T.T.H.P. and T.T.N.T. contributed to protein 
538 production and crystallization. A.D. performed the nanobodies selection and characterization.

539 E.D. provided the TssKFG clone. C.K. performed the BLI and HPLC experiments. L.L.,

540 Y.C., A.Z. and E.C performed the double hybrid and co-immunoprecipitation experiments.

\section{$542 \quad$ Funding information}

543 This work was supported by the Centre National de la Recherche Scientifique and the Aix-

544 Marseille Université, and grants from the Agence Nationale de la Recherche (ANR-14-CE14-

545 0006-02) and the French Infrastructure for Integrated Structural Biology (FRISBI). V.S.N

546 was supported by a fellowship from the French Embassy in Vietnam. L.L. and A.Z. were

547 supported by doctoral fellowships of the Ministère Français de l'Enseignement Supérieur et de

548 la Recherche and end-of-thesis fellowships from the Fondation pour la Recherche Médicale

549 (FRM) (FDT20160435498 and FDT20140931060 respectively). T.T.N.T. was supported by a

550 grant from the Ministère des Affaires Etrangères - France ( $\left.\mathrm{N}^{\circ} 861733 \mathrm{C}\right)$. Y.C. is supported by

551 a doctoral school PhD fellowship from the FRM (ECO20160736014).

552

553 Correspondence and requests for materials should be addressed to E.C. and C.C.

554

555 Acknowledgements

556 We thank the members of the Cambillau/Roussel and Cascales laboratories for insightful

557 discussions, Amel Kassa for initial work on TssK, Laure Journet for critical reading of the

558 manuscript, the Soleil (Saint Aubin, France) and ESRF (Grenoble, France) synchrotrons for

559 beam time allocation and Annick Brun, Isabelle Bringer and Olivier Uderso for technical

560 assistance.

561 


\section{References}

5631 Costa, T. R. et al. Secretion systems in Gram-negative bacteria: structural and

564 mechanistic insights. Nature reviews. Microbiology 13, 343-359,

565 doi:10.1038/nrmicro3456 (2015).

5662 Bonemann, G., Pietrosiuk, A. \& Mogk, A. Tubules and donuts: a type VI secretion

567 story. Molecular microbiology 76, 815-821, doi:10.1111/j.1365-2958.2010.07171.x

$568 \quad(2010)$

5693 Cascales, E. \& Cambillau, C. Structural biology of type VI secretion systems.

$570 \quad$ Philosophical transactions of the Royal Society of London. Series B, Biological

$571 \quad$ sciences 367, 1102-1111, doi:10.1098/rstb.2011.0209 (2012).

5724 Kapitein, N. \& Mogk, A. Deadly syringes: type VI secretion system activities in

573 pathogenicity and interbacterial competition. Current opinion in microbiology 16, 52-

$574 \quad$ 58, doi:10.1016/j.mib.2012.11.009 (2013).

5755 Zoued, A. et al. Architecture and assembly of the Type VI secretion system.

576 Biochimica et biophysica acta 1843, 1664-1673, doi:10.1016/j.bbamcr.2014.03.018

$577 \quad$ (2014).

5786 Ho, B. T., Dong, T. G. \& Mekalanos, J. J. A View to a Kill: The Bacterial Type VI

579 Secretion System. Cell host \& microbe 15, 9-21, doi:10.1016/j.chom.2013.11.008

$580 \quad$ (2014).

5817 Basler, M. Type VI secretion system: secretion by a contractile nanomachine.

582 Philosophical transactions of the Royal Society of London. Series B, Biological

$583 \quad$ sciences 370, doi:10.1098/rstb.2015.0021 (2015).

5848 Durand, E., Cambillau, C., Cascales, E. \& Journet, L. VgrG, Tae, Tle, and beyond: the 585 versatile arsenal of Type VI secretion effectors. Trends in microbiology 22, 498-507, 586 doi:10.1016/j.tim.2014.06.004 (2014). 
5879 Russell, A. B., Peterson, S. B. \& Mougous, J. D. Type VI secretion system effectors:

588 poisons with a purpose. Nature reviews. Microbiology 12, 137-148,

589 doi:10.1038/nrmicro3185 (2014).

59010 Kapitein, N. \& Mogk, A. Type VI secretion system helps find a niche. Cell host \&

$591 \quad$ microbe 16, 5-6, doi:10.1016/j.chom.2014.06.012 (2014).

$59211 \quad$ Wexler, A. G. et al. Human symbionts inject and neutralize antibacterial toxins to

593 persist in the gut. Proceedings of the National Academy of Sciences of the United

$594 \quad$ States of America 113, 3639-3644, doi:10.1073/pnas.1525637113 (2016).

59512 Chatzidaki-Livanis, M., Geva-Zatorsky, N. \& Comstock, L. E. Bacteroides fragilis

596 type VI secretion systems use novel effector and immunity proteins to antagonize

597 human gut Bacteroidales species. Proceedings of the National Academy of Sciences of

598 the United States of America 113, 3627-3632, doi:10.1073/pnas.1522510113 (2016).

59913 Sana, T. G. et al. Salmonella Typhimurium utilizes a T6SS-mediated antibacterial

600 weapon to establish in the host gut. Proceedings of the National Academy of Sciences

601 of the United States of America 113, E5044-5051, doi:10.1073/pnas.1608858113

$602 \quad$ (2016).

60314 Hecht, A. L. et al. Strain competition restricts colonization of an enteric pathogen and

604 prevents colitis. EMBO reports 17, 1281-1291, doi:10.15252/embr.201642282 (2016).

60515 Fu, Y., Waldor, M. K. \& Mekalanos, J. J. Tn-Seq analysis of Vibrio cholerae intestinal

606 colonization reveals a role for T6SS-mediated antibacterial activity in the host. Cell

607 host \& microbe 14, 652-663, doi:10.1016/j.chom.2013.11.001 (2013).

60816 Ma, L. S., Hachani, A., Lin, J. S., Filloux, A. \& Lai, E. M. Agrobacterium tumefaciens

609 deploys a superfamily of type VI secretion DNase effectors as weapons for

610 interbacterial competition in planta. Cell host \& microbe 16, 94-104,

611 doi:10.1016/j.chom.2014.06.002 (2014). 
61217 Coulthurst, S. J. The Type VI secretion system - a widespread and versatile cell

613 targeting system. Research in microbiology 164, 640-654,

614 doi:10.1016/j.resmic.2013.03.017 (2013).

61518 Bingle, L. E., Bailey, C. M. \& Pallen, M. J. Type VI secretion: a beginner's guide.

616 Current opinion in microbiology 11, 3-8, doi:S1369-5274(08)00007-6 (2008).

61719 Cascales, E. The type VI secretion toolkit. EMBO reports 9, 735-741,

618 doi:10.1038/embor.2008.131 (2008).

61920 Aschtgen, M. S., Bernard, C. S., De Bentzmann, S., Lloubes, R. \& Cascales, E. SciN

620 is an outer membrane lipoprotein required for type VI secretion in enteroaggregative

621 Escherichia coli. Journal of bacteriology 190, 7523-7531, doi:10.1128/JB.00945-08

$622 \quad(2008)$.

62321 Aschtgen, M. S., Gavioli, M., Dessen, A., Lloubes, R. \& Cascales, E. The SciZ

624 protein anchors the enteroaggregative Escherichia coli Type VI secretion system to the $625 \quad$ cell wall. Molecular microbiology, doi:10.1111/j.1365-2958.2010.07028.x (2010).

62622 Ma, L. S., Lin, J. S. \& Lai, E. M. An IcmF family protein, ImpLM, is an integral inner 627 membrane protein interacting with ImpKL, and its walker a motif is required for type 628 VI secretion system-mediated Hcp secretion in Agrobacterium tumefaciens. Journal of 629 bacteriology 191, 4316-4329, doi:10.1128/JB.00029-09 (2009).

63023 Felisberto-Rodrigues, C. et al. Towards a structural comprehension of bacterial type 631 VI secretion systems: characterization of the TssJ-TssM complex of an Escherichia 632 coli pathovar. PLoS pathogens 7, e1002386, doi:10.1371/journal.ppat.1002386 $633 \quad$ (2011).

63424 Aschtgen, M. S., Zoued, A., Lloubes, R., Journet, L. \& Cascales, E. The C-tail 635 anchored TssL subunit, an essential protein of the enteroaggregative Escherichia coli 
Sci-1 Type VI secretion system, is inserted by YidC. MicrobiologyOpen 1, 71-82,

637 doi:10.1002/mbo3.9 (2012).

63825 Durand, E. et al. Structural characterization and oligomerization of the TssL protein, a 639 component shared by bacterial type VI and type IVb secretion systems. The Journal of $640 \quad$ biological chemistry 287, 14157-14168, doi:10.1074/jbc.M111.338731 (2012).

64126 Durand, E. et al. Biogenesis and structure of a type VI secretion membrane core $642 \quad$ complex. Nature 523, 555-560, doi:10.1038/nature14667 (2015).

64327 Aschtgen, M. S., Thomas, M. S. \& Cascales, E. Anchoring the type VI secretion 644 system to the peptidoglycan: TssL, TagL, TagP... what else? Virulence 1, 535-540 $645 \quad$ (2010).

64628 Weber, B. S. et al. Genetic Dissection of the Type VI Secretion System in 647 Acinetobacter and Identification of a Novel Peptidoglycan Hydrolase, TagX, Required 648 for Its Biogenesis. mBio 7, doi:10.1128/mBio.01253-16 (2016).

64929 Santin, Y. G. \& Cascales, E. Domestication of a housekeeping transglycosylase for $650 \quad$ assembly of a Type VI secretion system. EMBO reports 18, 138-149, 651 doi:10.15252/embr.201643206 (2017).

65230 Leiman, P. G. et al. Type VI secretion apparatus and phage tail-associated protein 653 complexes share a common evolutionary origin. Proceedings of the National Academy $654 \quad$ of Sciences of the United States of America 106, 4154-4159, 655 doi:10.1073/pnas.0813360106 (2009).

65631 Brunet, Y. R., Henin, J., Celia, H. \& Cascales, E. Type VI secretion and bacteriophage 657 tail tubes share a common assembly pathway. EMBO reports, 658 doi:10.1002/embr.201337936(2014).

65932 Kudryashev, M. et al. Structure of the type VI secretion system contractile sheath. $660 \quad$ Cell 160, 952-962, doi:10.1016/j.cell.2015.01.037 (2015). 
66133 English, G., Byron, O., Cianfanelli, F. R., Prescott, A. R. \& Coulthurst, S. J.

662 Biochemical analysis of TssK, a core component of the bacterial Type VI secretion

663 system, reveals distinct oligomeric states of TssK and identifies a TssK-TssFG sub-

664 complex. The Biochemical journal, doi:10.1042/BJ20131426 (2014).

66534 Brunet, Y. R., Zoued, A., Boyer, F., Douzi, B. \& Cascales, E. The Type VI Secretion

666 TssEFGK-VgrG Phage-Like Baseplate Is Recruited to the TssJLM Membrane

667 Complex via Multiple Contacts and Serves As Assembly Platform for Tail

668 Tube/Sheath Polymerization. PLoS genetics 11, e1005545,

669 doi:10.1371/journal.pgen.1005545 (2015).

67035 Zoued, A. et al. Priming and polymerization of a bacterial contractile tail structure.

$671 \quad$ Nature 531, 59-63, doi:10.1038/nature17182 (2016).

67236 Boyer, F., Fichant, G., Berthod, J., Vandenbrouck, Y. \& Attree, I. Dissecting the

673 bacterial type VI secretion system by a genome wide in silico analysis: what can be

674 learned from available microbial genomic resources? BMC genomics 10, 104,

675 doi:10.1186/1471-2164-10-104 (2009).

67637 Pell, L. G., Kanelis, V., Donaldson, L. W., Howell, P. L. \& Davidson, A. R. The

677 phage lambda major tail protein structure reveals a common evolution for long-tailed

678 phages and the type VI bacterial secretion system. Proceedings of the National

679 Academy of Sciences of the United States of America 106, 4160-4165,

680 doi:0900044106 (2009).

68138 Kube, S. et al. Structure of the VipA/B type VI secretion complex suggests a

682 contraction-state-specific recycling mechanism. Cell reports $\mathbf{8}, 20-30$,

683 doi:10.1016/j.celrep.2014.05.034 (2014). 
68439 Basler, M., Pilhofer, M., Henderson, G. P., Jensen, G. J. \& Mekalanos, J. J. Type VI

685 secretion requires a dynamic contractile phage tail-like structure. Nature $\mathbf{4 8 3}$, 182-

$686 \quad$ 186, doi:10.1038/nature10846 (2012).

68740 LeRoux, M. et al. Quantitative single-cell characterization of bacterial interactions

688 reveals type VI secretion is a double-edged sword. Proceedings of the National

689 Academy of Sciences of the United States of America 109, 19804-19809,

$690 \quad$ doi:10.1073/pnas.1213963109(2012).

$69141 \quad$ Basler, M., Ho, B. T. \& Mekalanos, J. J. Tit-for-tat: type VI secretion system

692 counterattack during bacterial cell-cell interactions. Cell 152, 884-894,

693 doi:10.1016/j.cell.2013.01.042 (2013).

69442 Brunet, Y. R., Espinosa, L., Harchouni, S., Mignot, T. \& Cascales, E. Imaging type VI

695 secretion-mediated bacterial killing. Cell reports 3, 36-41,

696 doi:10.1016/j.celrep.2012.11.027 (2013).

69743 Lossi, N. S., Dajani, R., Freemont, P. \& Filloux, A. Structure-function analysis of

698 HsiF, a gp25-like component of the type VI secretion system, in Pseudomonas

699 aeruginosa. Microbiology 157, 3292-3305, doi:10.1099/mic.0.051987-0 (2011).

70044 Planamente, S. et al. TssA forms a gp6-like ring attached to the type VI secretion

701 sheath. The EMBO journal 35, 1613-1627, doi:10.15252/embj.201694024 (2016).

70245 Taylor, N. M. et al. Structure of the T4 baseplate and its function in triggering sheath

$703 \quad$ contraction. Nature 533, 346-352, doi:10.1038/nature17971 (2016).

70446 Leiman, P. G. et al. Morphogenesis of the T4 tail and tail fibers. Virology journal 7, $705 \quad 355$, doi:10.1186/1743-422X-7-355 (2010).

70647 Leiman, P. G. \& Shneider, M. M. Contractile tail machines of bacteriophages.

707 Advances in experimental medicine and biology 726, 93-114, doi:10.1007/978-1-

708 4614-0980-9_5(2012). 
70948 Zoued, A. et al. TssK is a trimeric cytoplasmic protein interacting with components of

710 both phage-like and membrane anchoring complexes of the Type VI secretion system.

711 The Journal of biological chemistry 288 27031-27041, doi:10.1074/jbc.M113.499772

$712 \quad$ (2013).

71349 Logger, L., Aschtgen, M. S., Guerin, M., Cascales, E. \& Durand, E. Molecular

714 Dissection of the Interface between the Type VI Secretion TssM Cytoplasmic Domain

715 and the TssG Baseplate Component. J Mol Biol 428, 4424-4437,

716 doi:10.1016/j.jmb.2016.08.032 (2016).

71750 Desmyter, A., Spinelli,S., Roussel,A. and Cambillau,C. Camelid nanobodies: killing

718 two birds with one stone. Curr. Opin. Struct. Biol. 32 1-8 (2015).

71951 Nguyen, V. S. et al. Inhibition of type VI secretion by an anti-TssM llama nanobody.

$720 \quad$ PloS one 10, e0122187, doi:10.1371/journal.pone.0122187 (2015).

$72152 \quad$ Nguyen, V. S. et al. Production, crystallization and X-ray diffraction analysis of a

722 complex between a fragment of the TssM T6SS protein and a camelid nanobody. Acta

723 crystallographica. Section F, Structural biology communications 71, 266-271,

724 doi:10.1107/S2053230X15000709 (2015).

72553 Desmyter, A. et al. Three Camelid VHH domains in complex with porcine pancreatic

726 alpha-amylase - Inhibition and versatility of binding topology. Journal of Biological

727 Chemistry 277, 23645-23650, doi:10.1074/jbc.M202327200 (2002).

72854 Spinelli, S., Tegoni, M., Frenken, L., van Vliet, C. \& Cambillau, C. Lateral

729 recognition of a dye hapten by a llama VHH domain. J Mol Biol 311, 123-129,

730 doi:10.1006/jmbi.2001.4856 (2001).

73155 Drozdetskiy, A., Cole, C., Procter, J. \& Barton, G. J. JPred4: a protein secondary

732 structure prediction server. Nucleic acids research 43, W389-394,

733 doi:10.1093/nar/gkv332 (2015). 
73456 Pettersen, E. F. et al. UCSF Chimera--a visualization system for exploratory research

735 and analysis. J Comput Chem 25, 1605-1612, doi:10.1002/jcc.20084 (2004).

$73657 \quad$ Farenc, C. et al. Molecular insights on the recognition of a Lactococcus lactis cell wall

737 pellicle by phage 1358 receptor binding protein. Journal of virology,

738 doi:10.1128/JVI.00739-14 (2014).

$73958 \quad$ Spinelli, S. et al. Lactococcal bacteriophage p2 receptor-binding protein structure

740 suggests a common ancestor gene with bacterial and mammalian viruses. Nature

$741 \quad$ structural \& molecular biology 13, 85-89, doi:nsmb1029 (2006).

74259 Ray, S. P. et al. Structural and biochemical characterization of human

743 adenylosuccinate lyase (ADSL) and the R303C ADSL deficiency-associated mutation.

744 Biochemistry 51, 6701-6713, doi:10.1021/bi300796y (2012).

74560 Casabona, M. G., Vandenbrouck, Y., Attree, I. \& Coute, Y. Proteomic

746 characterization of Pseudomonas aeruginosa PAO1 inner membrane. Proteomics 13,

747 2419-2423, doi:10.1002/pmic.201200565 (2013).

$74861 \quad$ Buttner, C. R., Wu, Y., Maxwell, K. L. \& Davidson, A. R. Baseplate assembly of

749 phage Mu: Defining the conserved core components of contractile-tailed phages and

750 related bacterial systems. Proceedings of the National Academy of Sciences of the

$751 \quad$ United States of America 113, 10174-10179, doi:10.1073/pnas.1607966113 (2016).

75262 Spinelli, S. et al. Modular structure of the receptor binding proteins of Lactococcus

753 lactis phages. The RBP structure of the temperate phage TP901-1. The Journal of

754 biological chemistry 281, 14256-14262 (2006).

75563 Casjens, S. R. \& Molineux, I. J. Short noncontractile tail machines: adsorption and

756 DNA delivery by podoviruses. Advances in experimental medicine and biology $\mathbf{7 2 6}$,

757 143-179, doi:10.1007/978-1-4614-0980-9_7 (2012). 
75864 Spinelli, S., Veesler, D., Bebeacua, C. \& Cambillau, C. Structures and host-adhesion

759 mechanisms of lactococcal siphophages. Frontiers in microbiology 5, 3,

$760 \quad$ doi:10.3389/fmicb.2014.00003 (2014).

76165 Sciara, G. et al. Structure of lactococcal phage p2 baseplate and its mechanism of

762 activation. Proceedings of the National Academy of Sciences of the United States of

$763 \quad$ America 107, 6852-6857, doi:1000232107 (2010).

76466 Zoued, A. et al. Structure-Function Analysis of the TssL Cytoplasmic Domain

765 Reveals a New Interaction between the Type VI Secretion Baseplate and Membrane

766 Complexes. J Mol Biol 428, 4413-4423, doi:10.1016/j.jmb.2016.08.030 (2016).

76767 van den Ent, F. \& Lowe, J. RF cloning: a restriction-free method for inserting target

768 genes into plasmids. Journal of biochemical and biophysical methods 67, 67-74,

769 doi:10.1016/j.jbbm.2005.12.008 (2006).

77068 Karimova, G., Pidoux, J., Ullmann, A. \& Ladant, D. A bacterial two-hybrid system

771 based on a reconstituted signal transduction pathway. Proceedings of the National

$772 \quad$ Academy of Sciences of the United States of America 95, 5752-5756 (1998).

77369 Battesti, A. \& Bouveret, E. The bacterial two-hybrid system based on adenylate

$774 \quad$ cyclase reconstitution in Escherichia coli. Methods 58, 325-334,

775 doi:10.1016/j.ymeth.2012.07.018 (2012).

$77670 \quad$ Pardon, E. et al. A general protocol for the generation of Nanobodies for structural

777 biology. Nature protocols 9, 674-693, doi:10.1038/nprot.2014.039 (2014).

77871 Arbabi Ghahroudi, M., Desmyter, A., Wyns, L., Hamers, R. \& Muyldermans, S.

779 Selection and identification of single domain antibody fragments from camel heavy-

$780 \quad$ chain antibodies. FEBS letters 414, 521-526 (1997). 
78172 Desmyter, A. et al. Viral infection modulation and neutralization by camelid

782 nanobodies. Proceedings of the National Academy of Sciences of the United States of

$783 \quad$ America 110, E1371-1379, doi:10.1073/pnas.1301336110 (2013).

$78473 \quad$ Kabsch, W. Xds. Acta Crystallogr D Biol Crystallogr 66, 125-132,

785 doi:S0907444909047337 (2010).

78674 Vagin, A. \& Teplyakov, A. Molecular replacement with MOLREP. Acta Crystallogr

787 D Biol Crystallogr 66, 22-25, doi:10.1107/S0907444909042589 (2010).

$78875 \quad$ Blanc, E. et al. Refinement of severely incomplete structures with maximum

789 likelihood in BUSTER-TNT. Acta Crystallogr D Biol Crystallogr 60, 2210-2221,

$790 \quad$ doi:S0907444904016427 (2004).

79176 Emsley, P., Lohkamp, B., Scott, W. G. \& Cowtan, K. Features and development of

792 Coot. Acta Crystallogr D Biol Crystallogr 66, 486-501,

793 doi:10.1107/S0907444910007493 (2010).

79477 Karplus, P. A. \& Diederichs, K. Linking crystallographic model and data quality.

$795 \quad$ Science 336, 1030-1033, doi:10.1126/science.1218231 (2012).

79678 Sheldrick, G. M. A short history of SHELX. Acta Crystallogr A 64, 112-122,

797 doi:10.1107/S0108767307043930 (2008).

79879 Pape, T. a. S., T.R. HKL2MAP: a graphical user interface for macromolecular phasing

799 with SHELX programs. J. Applied Crystallogr. 37, 853-844 (2004).

$80080 \quad$ McCoy, A. J. et al. Phaser crystallographic software. Journal of applied

$801 \quad$ crystallography 40, 658-674, doi:10.1107/S0021889807021206 (2007).

80281 Cowtan, K. Recent developments in classical density modification. Acta Crystallogr D

803 Biol Crystallogr 66, 470-478, doi:10.1107/S090744490903947X (2010). 
80482 Cowtan, K. The Buccaneer software for automated model building. 1. Tracing protein $805 \quad$ chains. Acta Crystallogr D Biol Crystallogr 62, 1002-1011, 806 doi:10.1107/S0907444906022116(2006).

807 
810 Figure 1. Structure of TssK. a, Ribbon view of the TssK trimer. Monomers A, B and C are

811 colored pink, blue and green, respectively. The location of the N-terminal (shoulder), central

812 (neck) and C-terminal (head) domains is indicated. b, Ribbon view of TssK monomer B (full-

813 length TssK). The monomer is rainbow-colored (blue to red from $\mathrm{N}$ - to C-terminal). The $\alpha$ -

814 helices and $\beta$-strands are numbered. $(\mathbf{c}, \mathbf{d})$ Side $(\mathbf{c})$ and bottom $(\mathbf{d})$ views of the TssK X-ray

815 structure fitted into the TssK negative-stain electron microscopy map (EMD-5739),

816 highlighting that the TssK C-terminal head domain is disordered.

818 Figure 2. Structure of the TssK $\mathrm{SN}_{\mathrm{SN}}-\mathbf{n b K 1 8 - n b K 2 7}$ complex. a, Ribbon view of the TssK $\mathrm{SN}^{-}$

819 nbK18-nbK27 complex. Monomers A, B and C are colored pink, blue and green,

820 respectively. The three nbK18 are colored grey and the unique nbK27 is colored beige. $\mathbf{b}$,

821 Same view as in (a), rotated by $90^{\circ}$ presenting the bottom of the $\mathrm{TssK}_{\mathrm{SN}}$ domain. c, Same

822 orientation and colors as in (b), but with a molecular surface representation. $\mathbf{d}$, Same

823 orientation as in (b). The $\mathrm{TssK}_{\mathrm{SN}}$ domain trimer (beige) and the nbK18 nanobody (grey) are

824 represented as ribbons. The $\mathrm{TssK}_{\mathrm{SN}} \mathrm{N}$-terminal segments ordered upon surface binding are

825 identified by their ribbon darker color (brown) and their green semi-transparent surface.

827 Figure 3. Structural comparison of the TssK N-terminal shoulder domain with shoulder 828 domains of siphophage receptor-binding proteins. Superimposed ribbon views of the TssK

829 N-terminal shoulder domain (pink) with the shoulder domains of phages p2 (green) and 1358

830 (blue) RBPs (PDB 1ZRU and 4L9B respectively). 
832 Figure 4. Protein-protein interaction study of TssK domains. a, Bacterial two-hybrid

833 assay. BTH101 reporter cells producing the indicated proteins or domains $\left(\mathrm{K}, \mathrm{TssK}\right.$; $\mathrm{K}_{\mathrm{SN}}$,

834 TssK shoulder and neck domains; $K_{S}$, TssK shoulder domain; $K_{N}$, TssK neck domain; $K_{H}$,

835 TssK head domain) fused to the T18 or T25 domain of the Bordetella adenylate cyclase were

836 spotted on X-Gal indicator plates. The blue color of the colony reflects the interaction

837 between the two proteins. TolB and Pal are two proteins known to interact but unrelated to the

838 T6SS. The BACTH experiments have been performed in triplicate with identical results. b,

839 Co-immunoprecipitation assay. A mixture of soluble lysates from $2 \times 10^{10}$ E. coli $\mathrm{K}-12$

840 W3110 cells producing VSV-G-tagged TssK shoulder + neck $\left(\mathrm{K}_{\mathrm{SN}}\right)$, TssK shoulder $\left(\mathrm{K}_{\mathrm{S}}\right)$ and

841 TssK head $\left(\mathrm{K}_{\mathrm{H}}\right)$ domains (individually shown on the left panel) was mixed with FLAG-

842 tagged TssF + TssG, TssL $_{\mathrm{C}}$ or TssM $\mathrm{C}$. FLAG-tagged proteins were subjected to

843 immunoprecipitation with anti-FLAG-coupled beads, and the immunoprecipitated material

844 was separated by $12.5 \%$ crylamide SDS-PAGE and immunodetected anti-VSV-G monoclonal

845 antibodies. The position of each protein and domain is indicated, Molecular weight markers

846 (in $\mathrm{kDa}$ ) are indicated on the right. The co-immunoprecipitation experiments have been

847 performed in duplicate with identical results. c, Gel filtration chromatograms of TssKFG

848 (brown), $\operatorname{TssK}_{\mathrm{SN}} \mathrm{FG}$ (red), $\mathrm{TssK}_{\mathrm{SN}} \mathrm{FG}+$ nbK18 (blue) and $\mathrm{TssK}_{\mathrm{SN}} \mathrm{FG}+$ nbK27 (green); inset:

849 enlargment of the top of the peaks; the elution time is indicated above each peak. d, SDS gels

850 of the main peak of TssKFG (brown) and $\mathrm{TssK}_{\mathrm{SN}} \mathrm{FG}$ (red) showing the presence of TssK,

851 TssF and TssG, and TssK $\mathrm{SN}_{\mathrm{SN}}$, TssF and TssG, respectively. e, SDS gels of the main peak of

$852 \mathrm{TssK}_{\mathrm{SN}} \mathrm{FG}\left(\right.$ red), $\mathrm{TssK}_{\mathrm{SN}} \mathrm{FG}+$ nbK18 (blue) and $\mathrm{TssK}_{\mathrm{SN}} \mathrm{FG}+$ nbK27 (green) showing the

853 presence of nbK18 attached to TssKSNFG, but not nbK27. a-b: The experiments were done

854 at least in triplicate and a representative result is shown. c-e: The experiments were done in

855 triplicate and a representative result is shown.

856 
857 Figure 5. Schematic model of the baseplate docked to the membrane complex,

858 highlighting the connector role of TssK. The TssJLM membrane complex is represented in

859 green whereas TssK is colored beige and the other baseplate components, TssFGE are colored

860 blue, VgrG is yellow and Hcp orange. The tail complex is shown in dark green. The three

861 domains of TssK are represented in beige, the N-terminal shoulder domain anchored to the

862 baseplate and the C-terminal head domain bound to the membrane complex. 

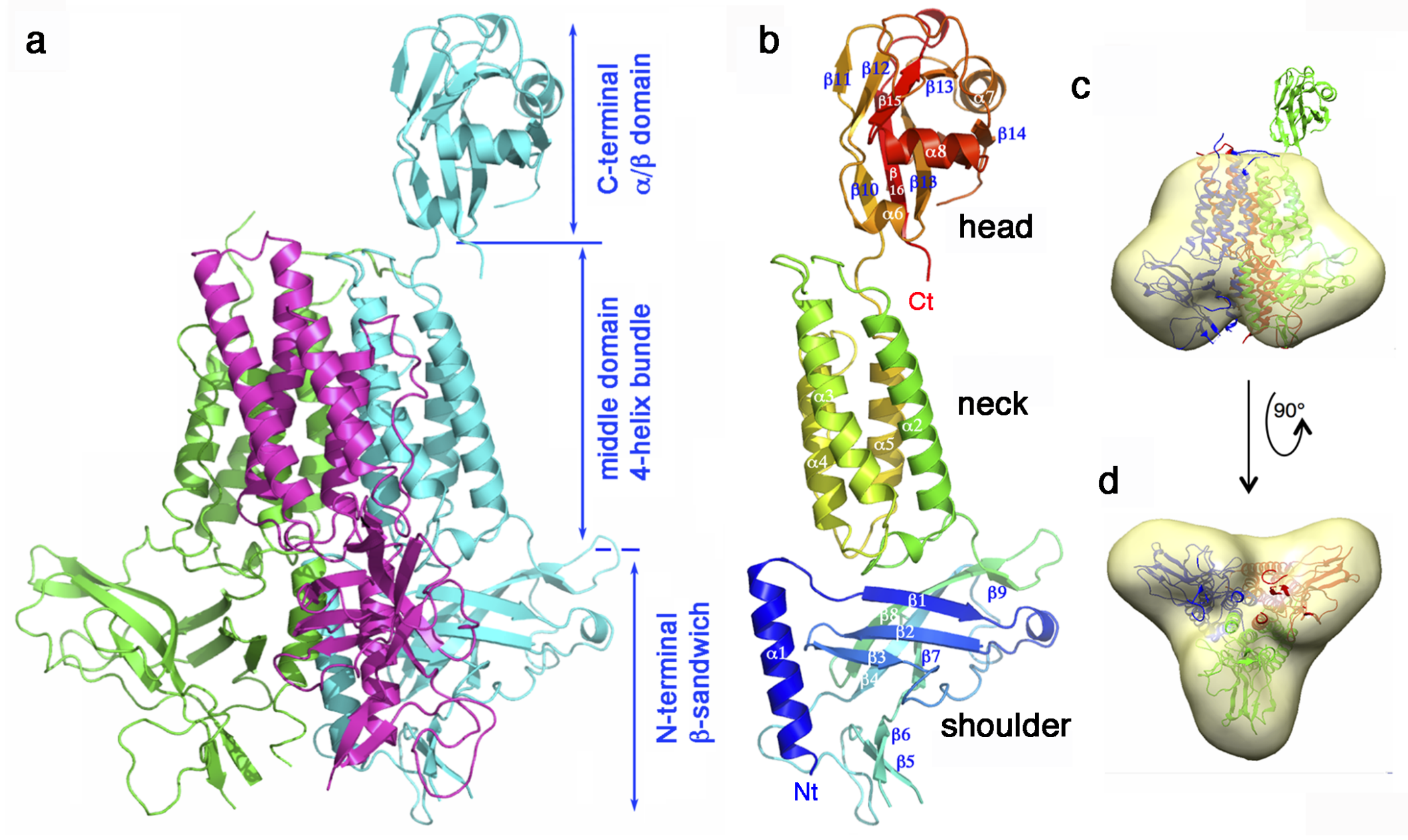


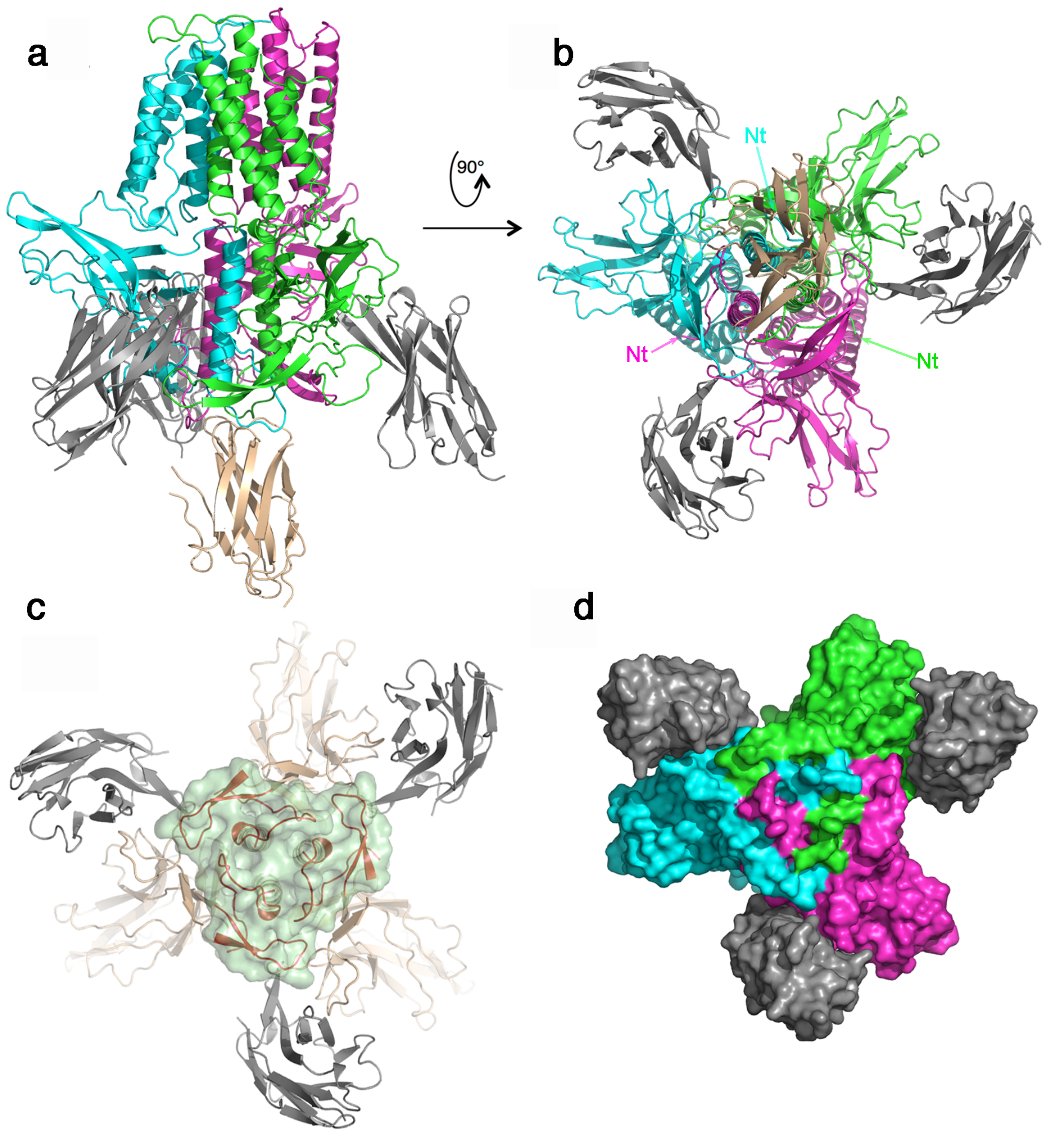




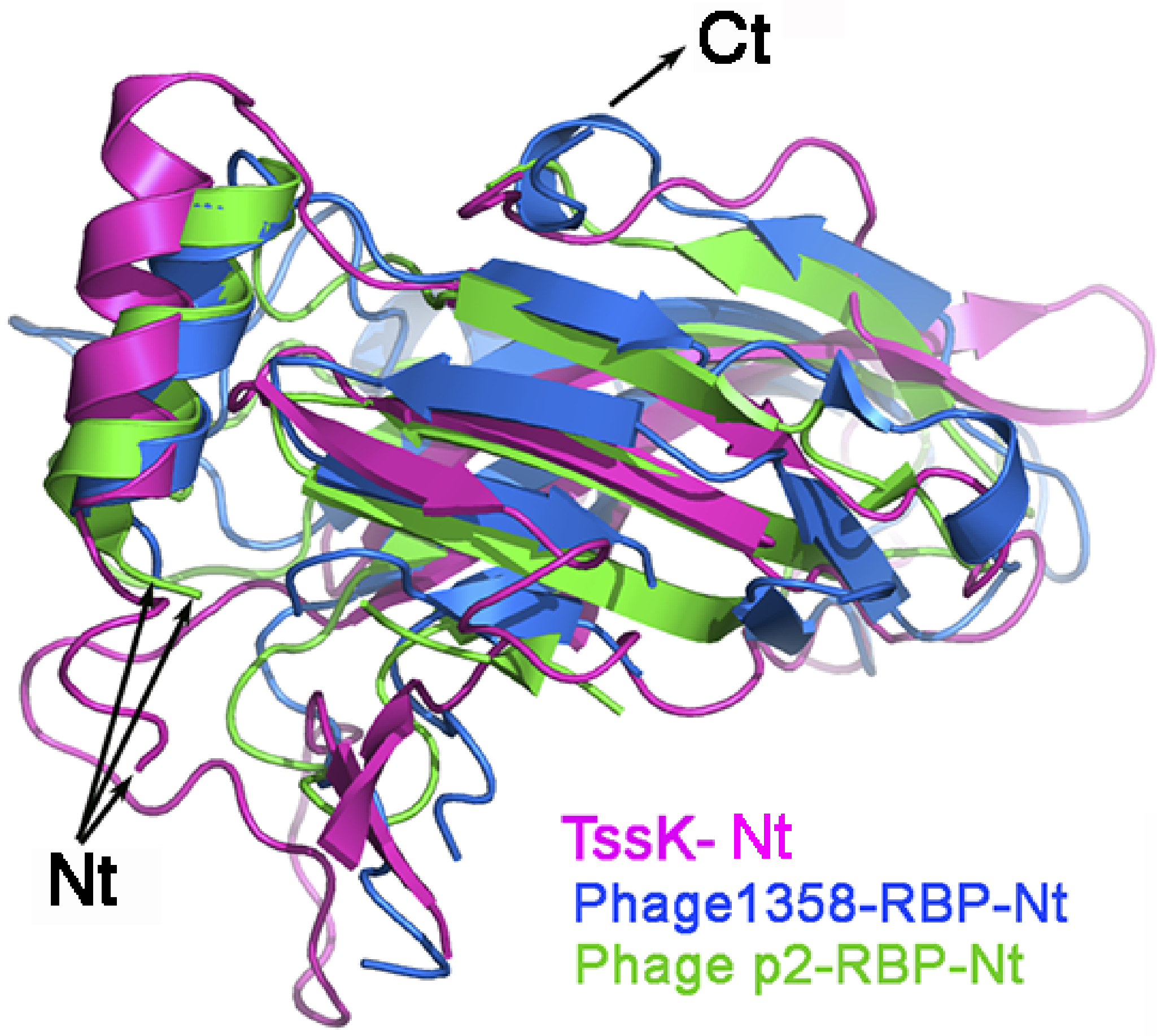




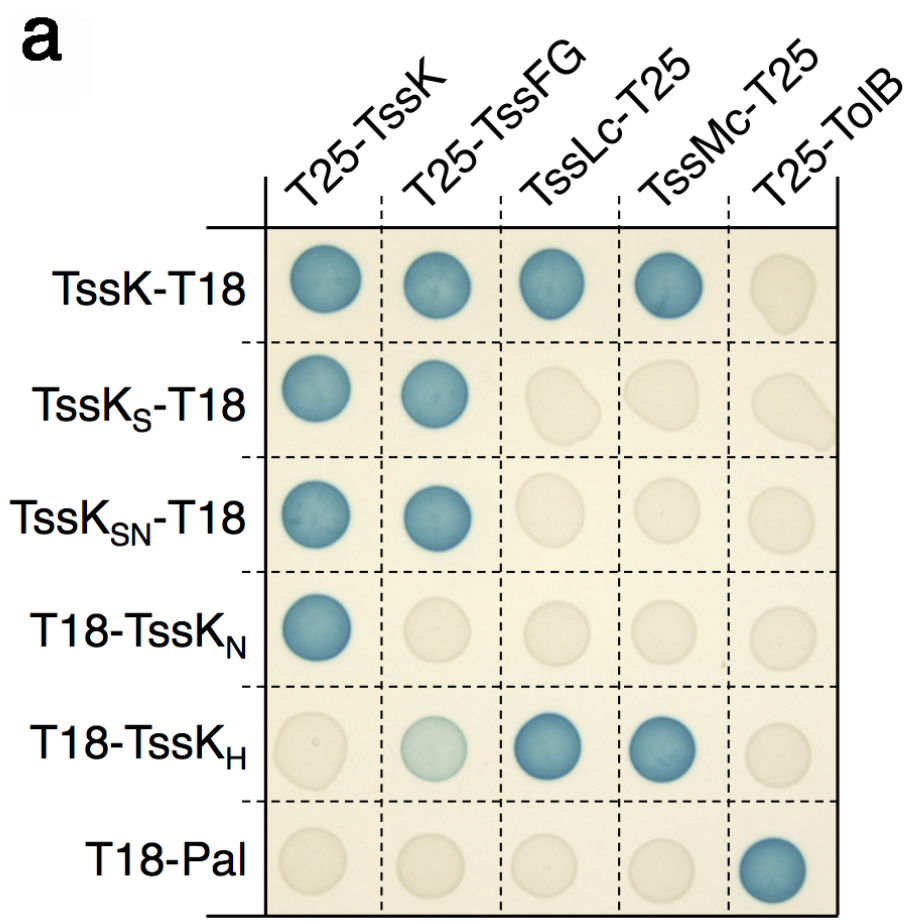

b

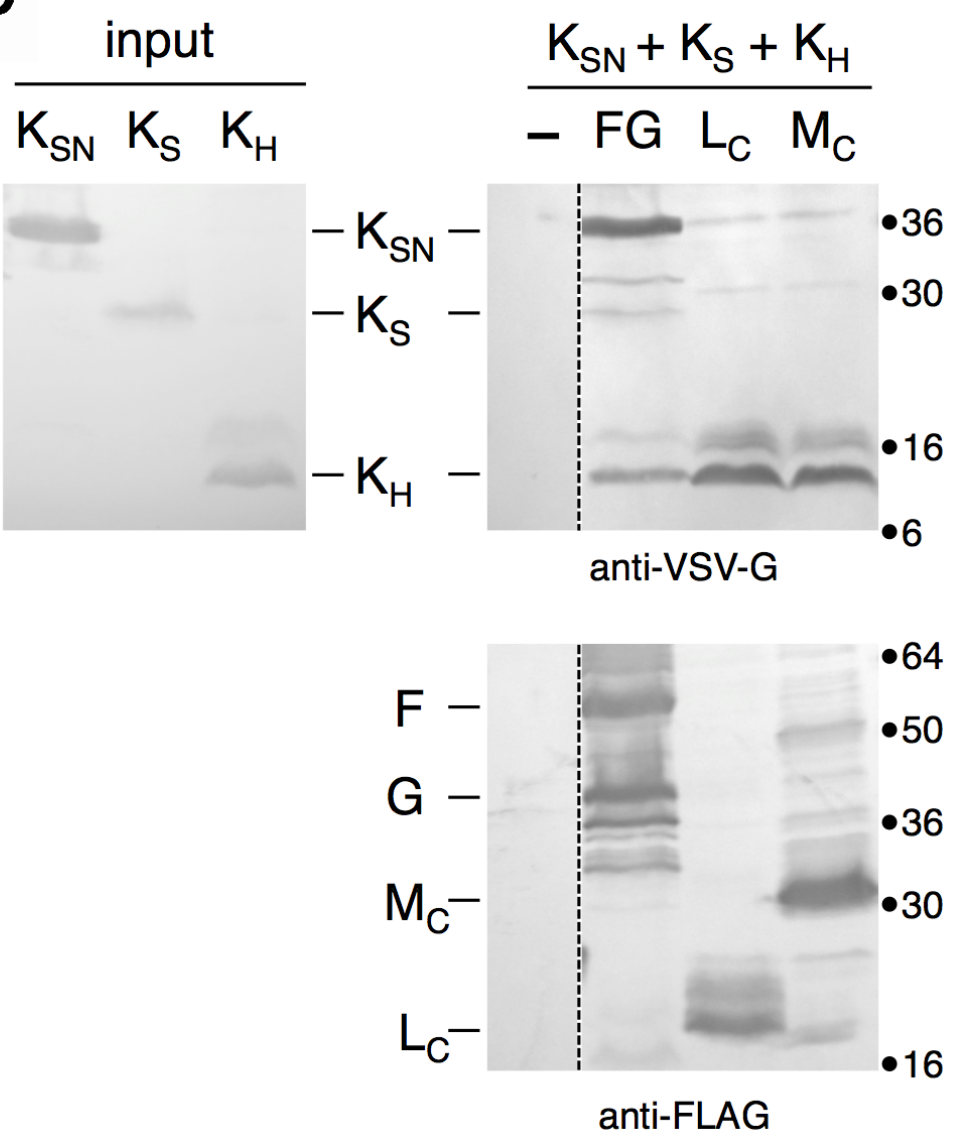

C






\section{OM}

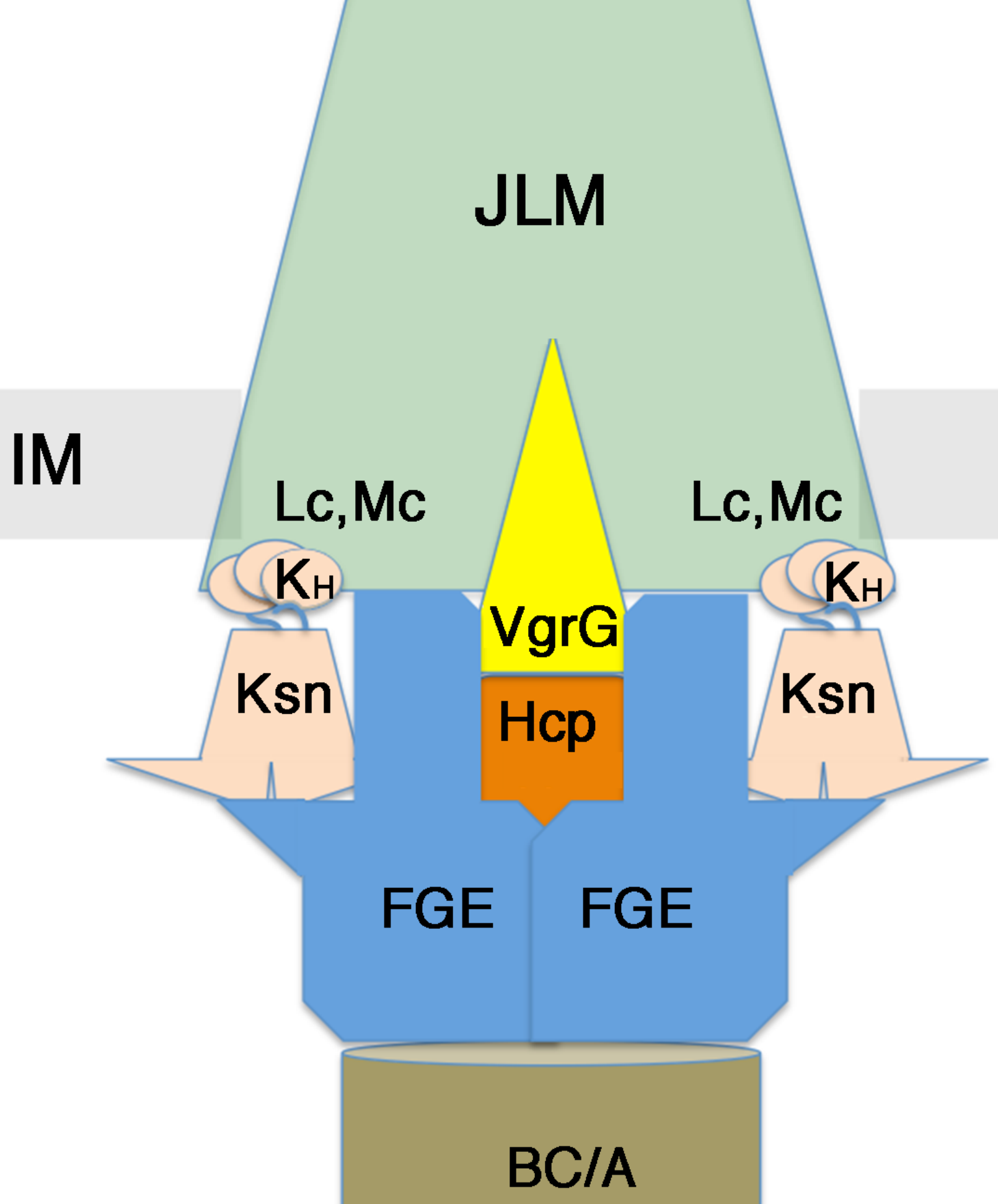


Type VI secretion TssK baseplate protein exhibits structural similarities with phage receptor binding protein and evolved to bind the membrane complex

Van Son Nguyen, Laureen Logger, Silvia Spinelli, Pierre Legrand, Thi Thanh Huyen Pham, Thi Trang Nhung Trinh, Yassine Cherrak, Abdelrahim Zoued, Aline Desmyter, Eric Durand, Alain Roussel, Christine Kellenberger, Eric Cascales ${ }^{*}$ and Christian Cambillau*

- corresponding authors: Eric Cascales (cascales@imm.cnrs.fr) and Christian Cambillau (cambillau@afmb.univ-mrs.fr).

This PDF file includes:

Supplementary Figures 1-10

Supplementary Tables 1-3 
a
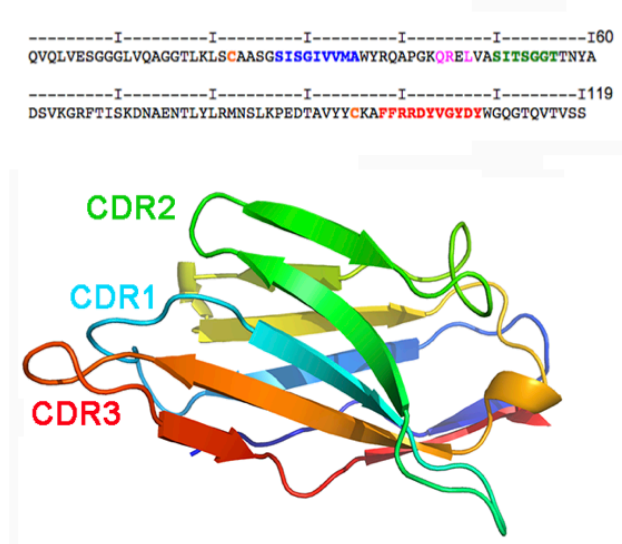

b

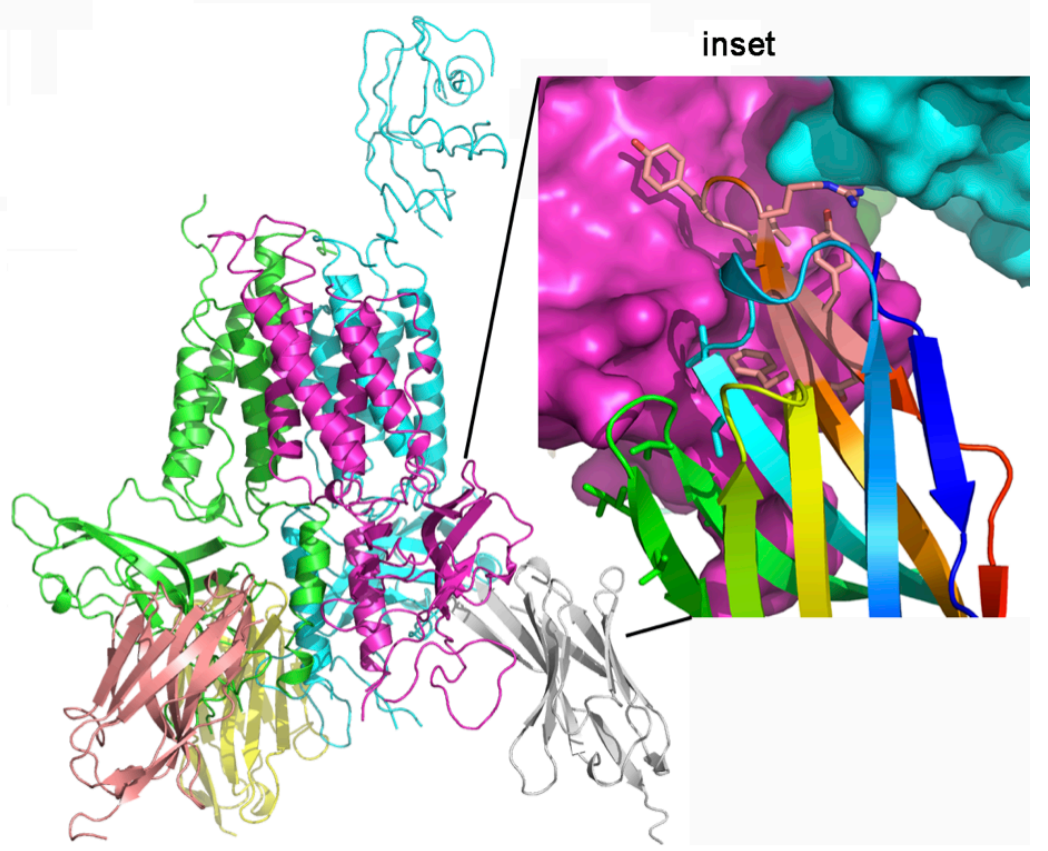

Supplementary Figure 1. Characterization of the nbK18 nanobody and its interaction with

TssK. a, sequence and structure of TssK-specific nbK18 llama single-chain antibody. The nbK18 sequence is presented on top of its rainbow-colored ribbon structure. The three complementarydetermining regions (CDR) are colored blue, green and red, respectively in the sequence and indicated with the same color code in the ribbon structure. b, structure of the trimeric TssK-nbK18 complex. Each TssK is colored as in Fig. 1A (monomers A, B and C in pink, blue and green, respectively) and the three nbK18 nanobodies, bound to TssK shoulder domain, are shown in grey, yellow and salmon, respectively. In the inset is shown a close-up of the TssK-nbK18 interaction at monomer A. TssK is shown in surface representation whereas nbK18 is shown in ribbon. The magnification highlights the side-chains of nbK18 that contact TssK. 
a

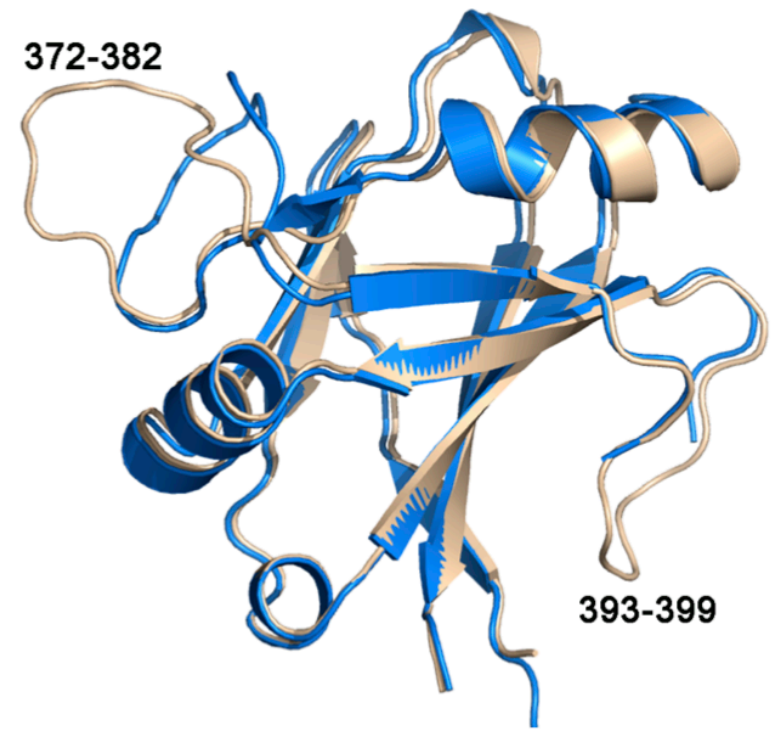

b

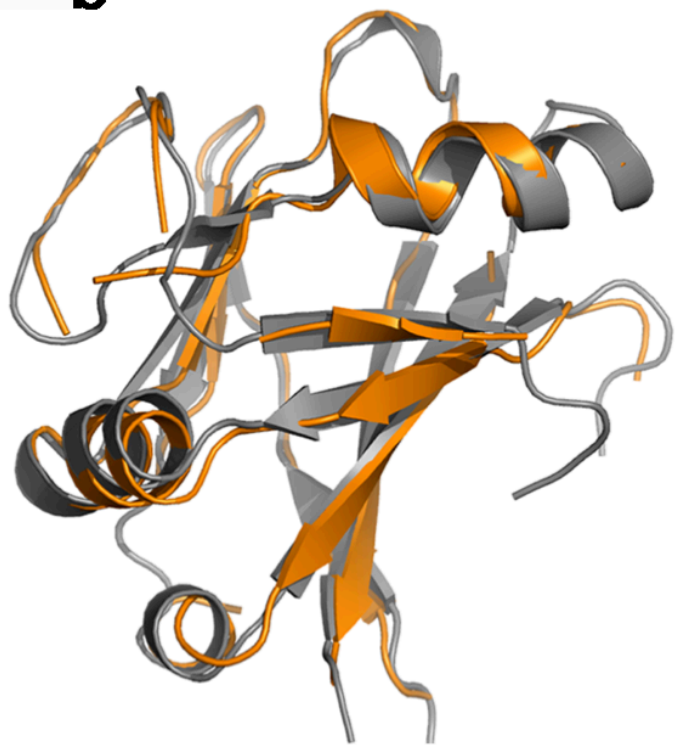

Supplementary Figure 2. Structural analysis of the TssK C-terminal domain. a, overlay between the TssK C-terminal head domain crystallized alone (beige) and in the refined full-length structure after insertion of the TssK C-terminal shoulder domain structure (blue). b, overlay between the TssK C-terminal head domain crystallized alone (grey) and in the full-length structure before the TssK C-terminal head structure insertion (orange). 


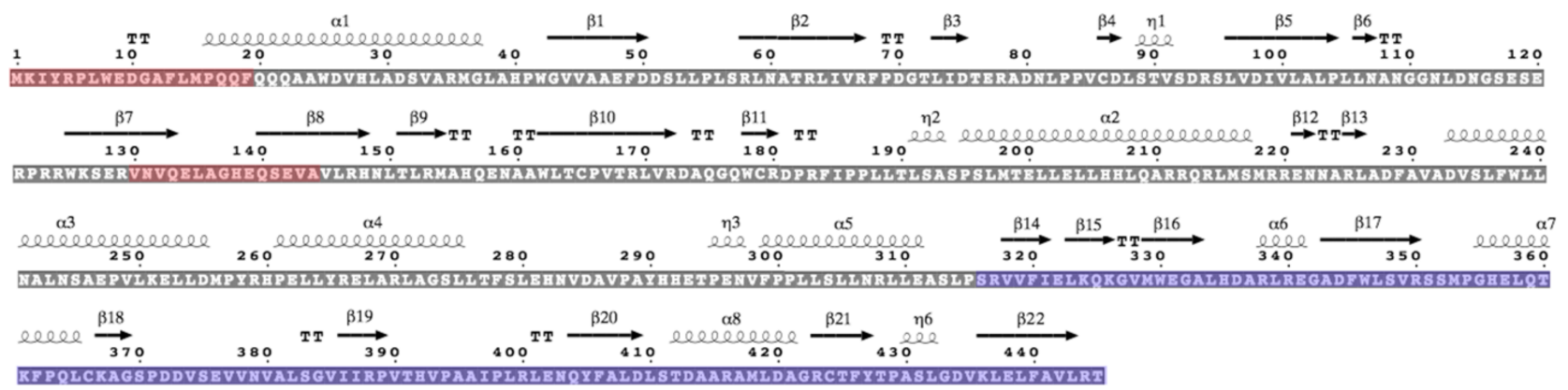



$\begin{array}{lll}\text { TssK+ nbK18 } & \text { GVv } & \text { GVI(subunit B only, high B-factors) } \\ \text { TssKsn } & \text { GVI } & \\ \text { TssKsN+ nbK18+nbK27 } & \text { RPI GVV }\end{array}$

Supplementary Figure 3. Linear sequence representation of TssK with secondary structures. The domains or segments present in the different crystal structures are identified with different colors. 




Supplementary Figure 4. Structural homologies of the TssK central domain (neck). Ribbon view of the overlay of $\mathrm{t} \mathrm{TssK}_{\mathrm{N}}$ helical bundle (rainbow colored) with the Human adenylosuccinate lyase (grey, PDB: 4FFX). 



shoulder

(1-193)

interaction TssFG with
$\mathbf{K}_{\mathbf{H}}$

$\mathrm{Ks}$

\section{Ks}

head

(315-447)

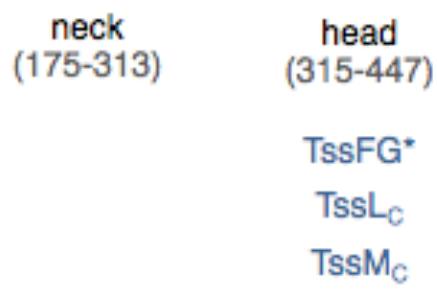

TSSFG TSSL ${ }_{C}$ TSSM



Supplementary Figure 5. Summary of the TssK domains interaction study. The different constructs used for the co-immunoprecipitation analyses are shown on left, with their boundaries (light green, N-terminal shoulder domain $\left(\mathrm{K}_{\mathrm{S}}\right)$, amino-acids 1-193; dark green, central neck domain $\left(\mathrm{K}_{\mathrm{N}}\right)$, amino-acids 175-313; blue, C-terminal head domain $\left(\mathrm{K}_{\mathrm{H}}\right)$, amino-acids 315-447). The interactions identified by co-immunoprecipitations are indicated with ' + ' on the right $(*$, interaction observed by co-immunoprecipitation but not confirmed by co-purification and nanobody binding assays). 

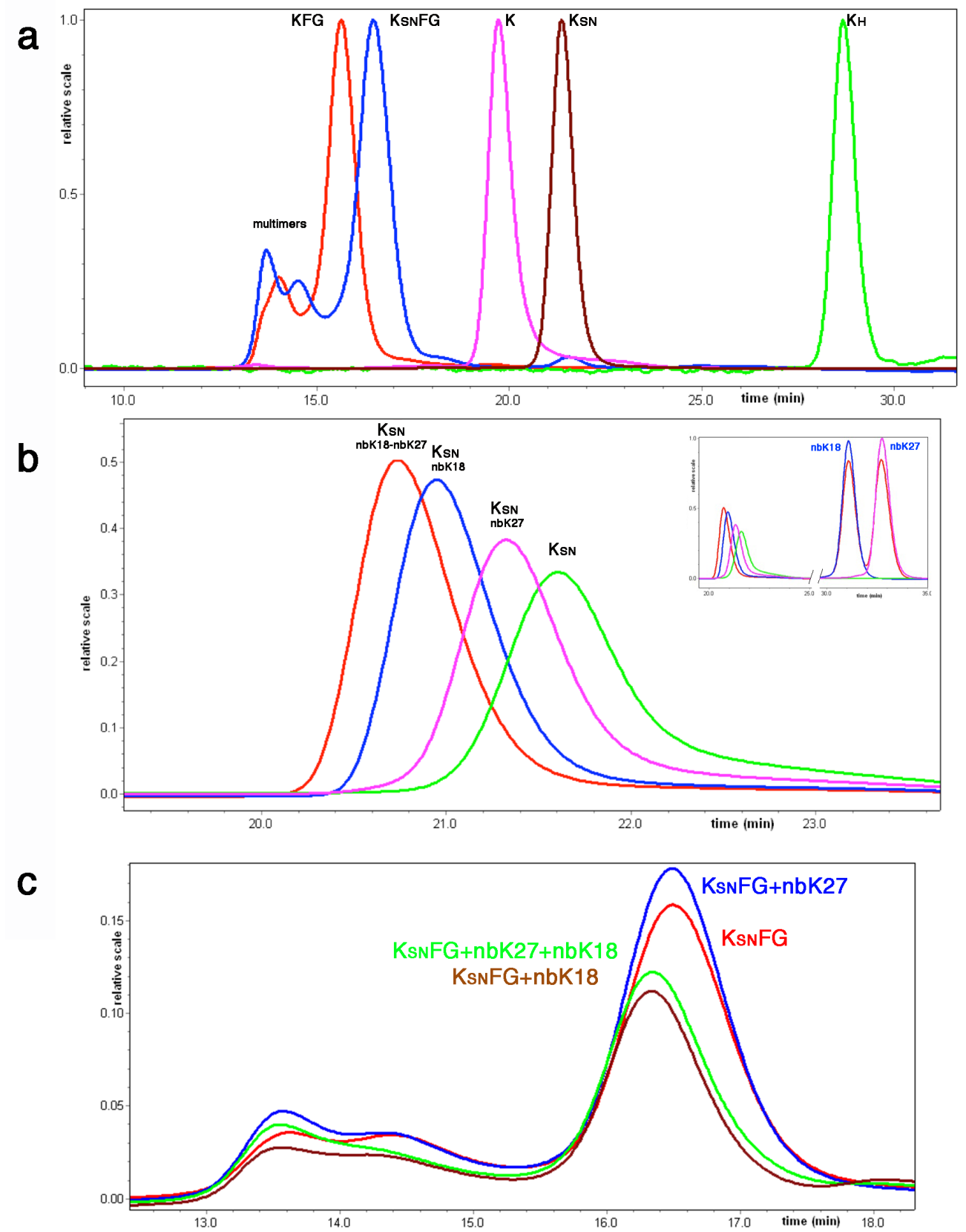

Supplementary Figure 6. a, HPLC gel Filtration chromatograms of TssKh, TssKsn, TssK, TssKsnFG and TssKFG. The peaks appearing before TssKFG are assigned to multimers. The peak amplitude has been scaled to 1.0. b, HPLC gel Filtration chromatograms of TssKSN in the presence of an excess of nbK18, nbK27 and nbK18, nbK27. Inset: the full chromatogram with the nanobodies. c, HPLC gel Filtration chromatograms of TssKSNFG alone or in the presence of an excess nbK27 of nbK18, and nbK $18+$ nbK27. a,c: the experiments were done in triplicate and a representative result is shown. 


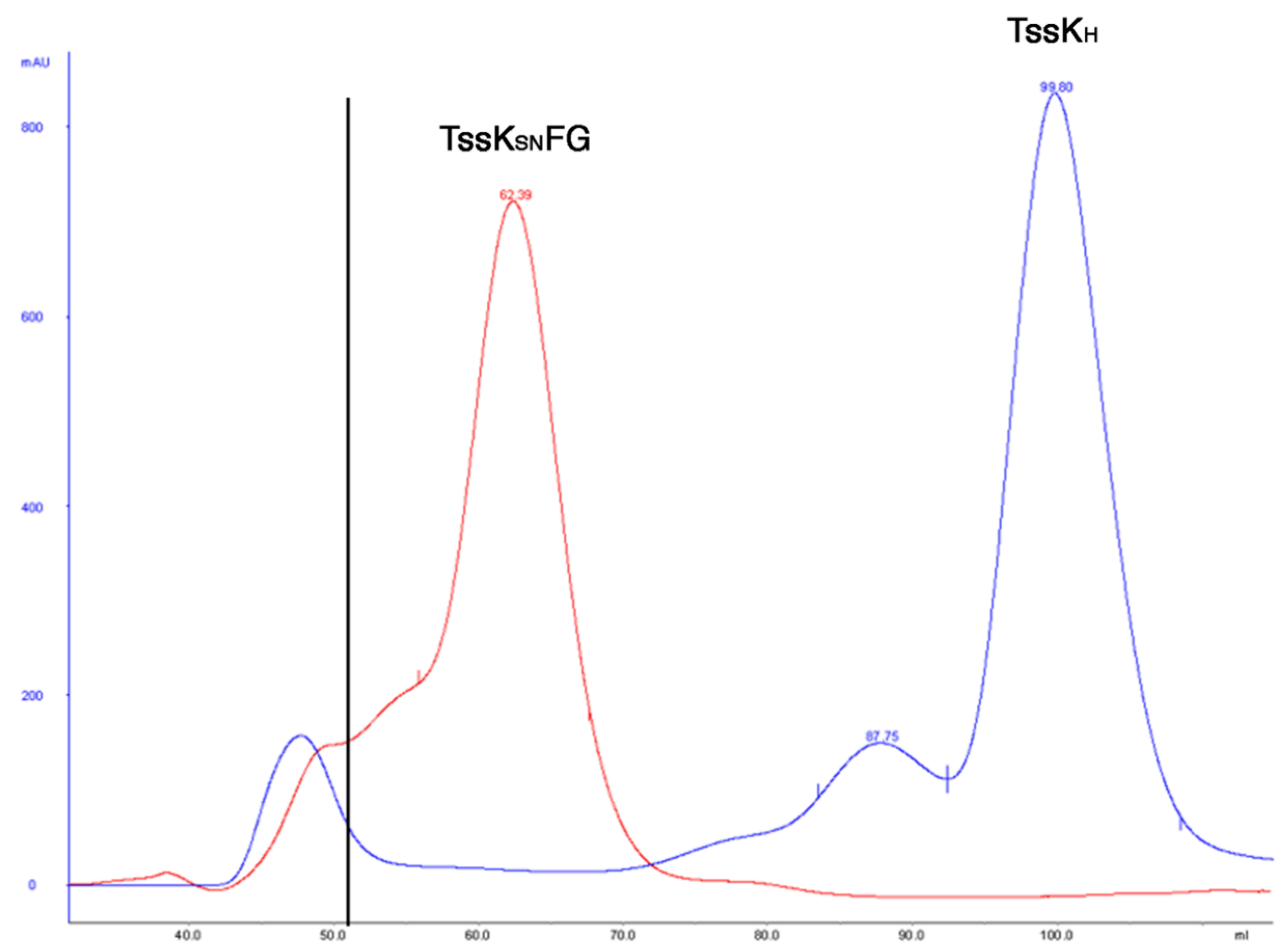

Supplementary Figure 7. Gel Filtration chromatograms of the product resulting from the $t s s K_{H} F G$ expression (blue) compared to the $t_{s s} K_{S N} F G$ expression (orange). The main peak of the blue chromatogram corresponds to TssKH alone, indicating that a stable TssKHFG complex is not formed. 


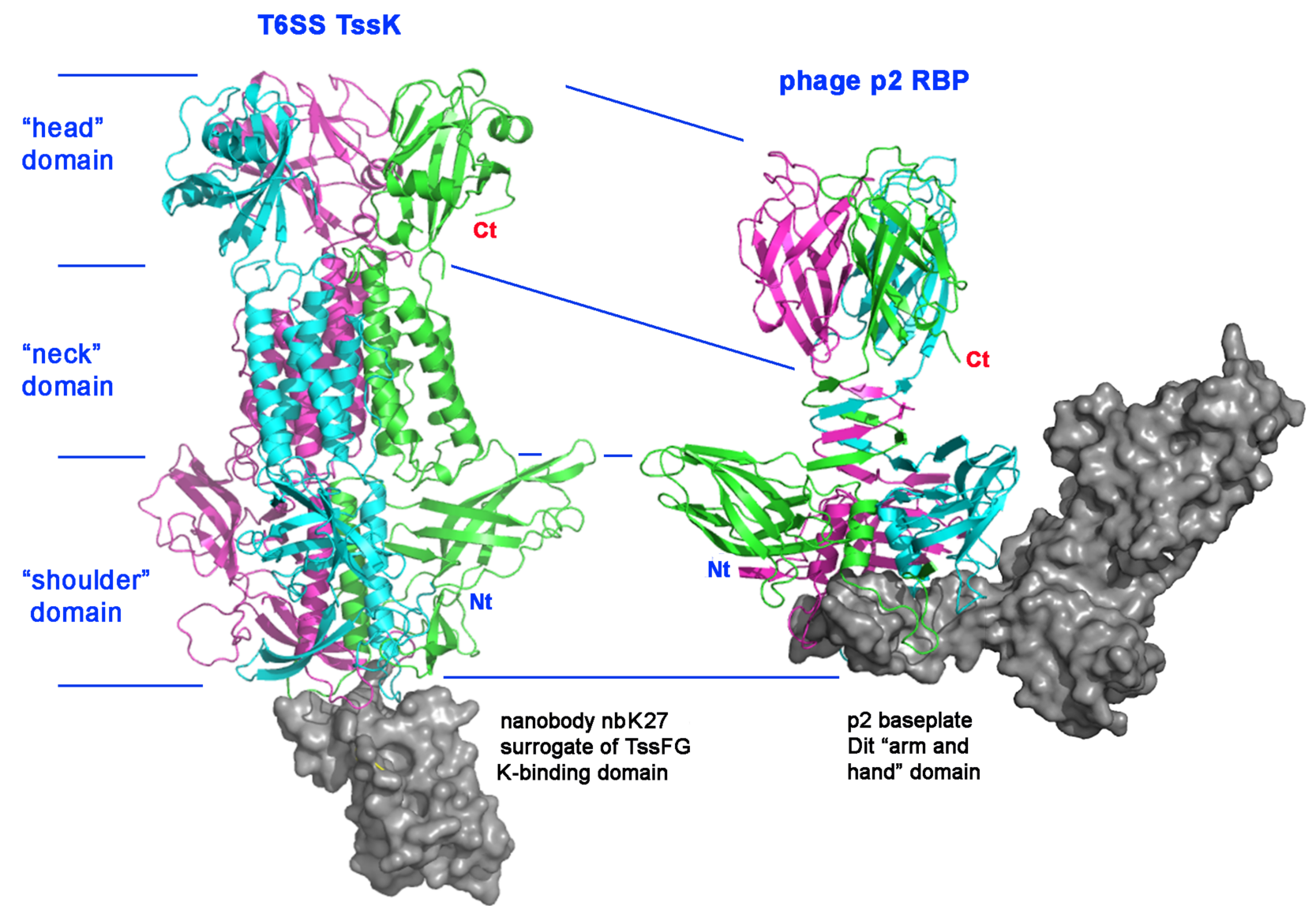

Supplementary Figure 8. Comparison of the TssK and phage p2 RBP structures evidencing the common topology of these trimers formed of shoulder, neck and head domains. The binding of TssK to nbK27 (that occupies the binding site of TssFG) is reminiscent of the binding of the Dit arm and hand extension of the phage p2 Dit molecule. 
a pCDF-Duet1 (TssKFG)

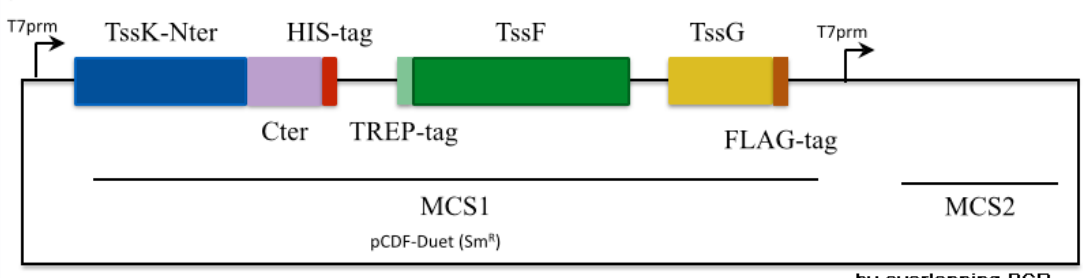

by overlapping PCR

b

pCDF-Duet1 (TssKFG) - STREP and FLAG tags delete

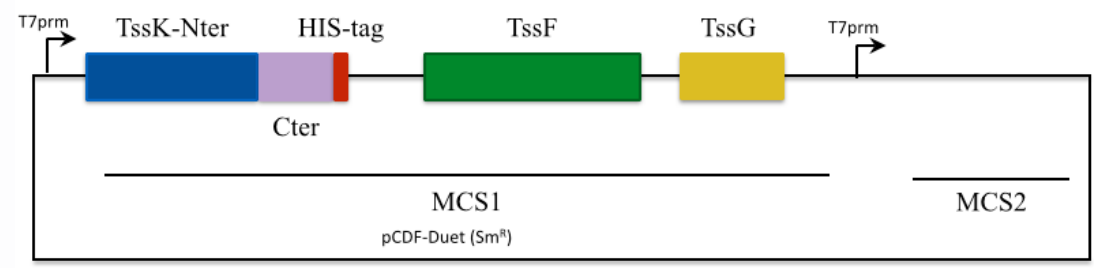

Del-STREP-pKFG-F: TGAGAATTCAAGGAGATATACATATGgacgatttaaccetgcgttatttg Del-STREP-pKFG-R: caaaataacgcagggttaaatcgtcCATATGTATATCTCCTTGAATTCTCA

Del-FLAG-pKFG-F: GGAAAATGGCGATTATCGCTGGtaatcgaagcttgcggcegc Del-FLAG-pKFG-R: gcggcegcaagcttcgattaCCAGCGATAATCGCCATTTTCC

c pCDF-Duet1 (TssK FG)

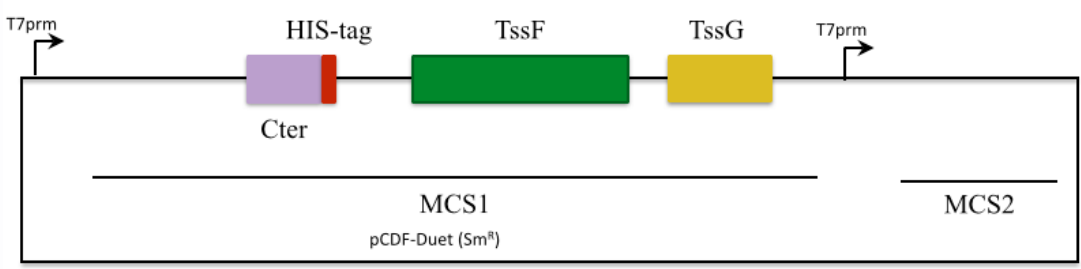

KFG-DelKNter-Gibson-F: GATATACATATGtcccgtgtggttttcattgaactgaaac

by Gibson assembly KFG-DelKNter-Gibson-R: cacacgggaCATATGTATATCTCCTTGGATCCTGGCTG

d pCDF-Duet1 (TssKntFG)

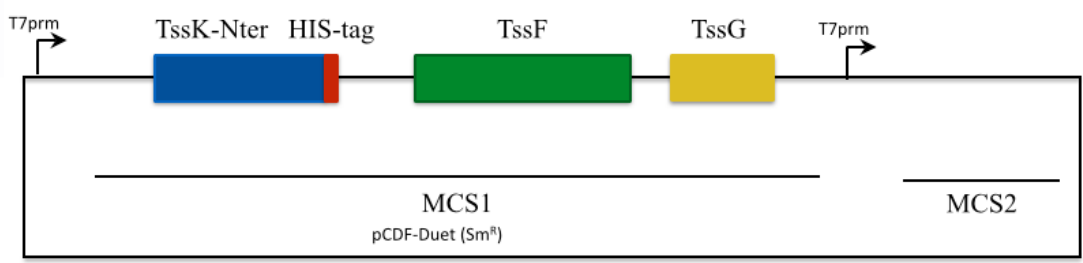

Primers: Delete TssK-Cter in pCDF-KFG

by Gibson assembly



Del-KCter-pKFG-Gib-R: TTCTCAGTGGTGATGATGGTGATGGGAAGGCAGGCTTGCCTCCAGC

Supplementary Figure 9. Schematic representation of the tssKFG His, strep and flag (a), tssKFG $H i s$ (b), $t s s K_{S N} F G$ (c) and $t s s K_{H} F G(\mathbf{d})$ constructs used in this study. 
Fig. 4b
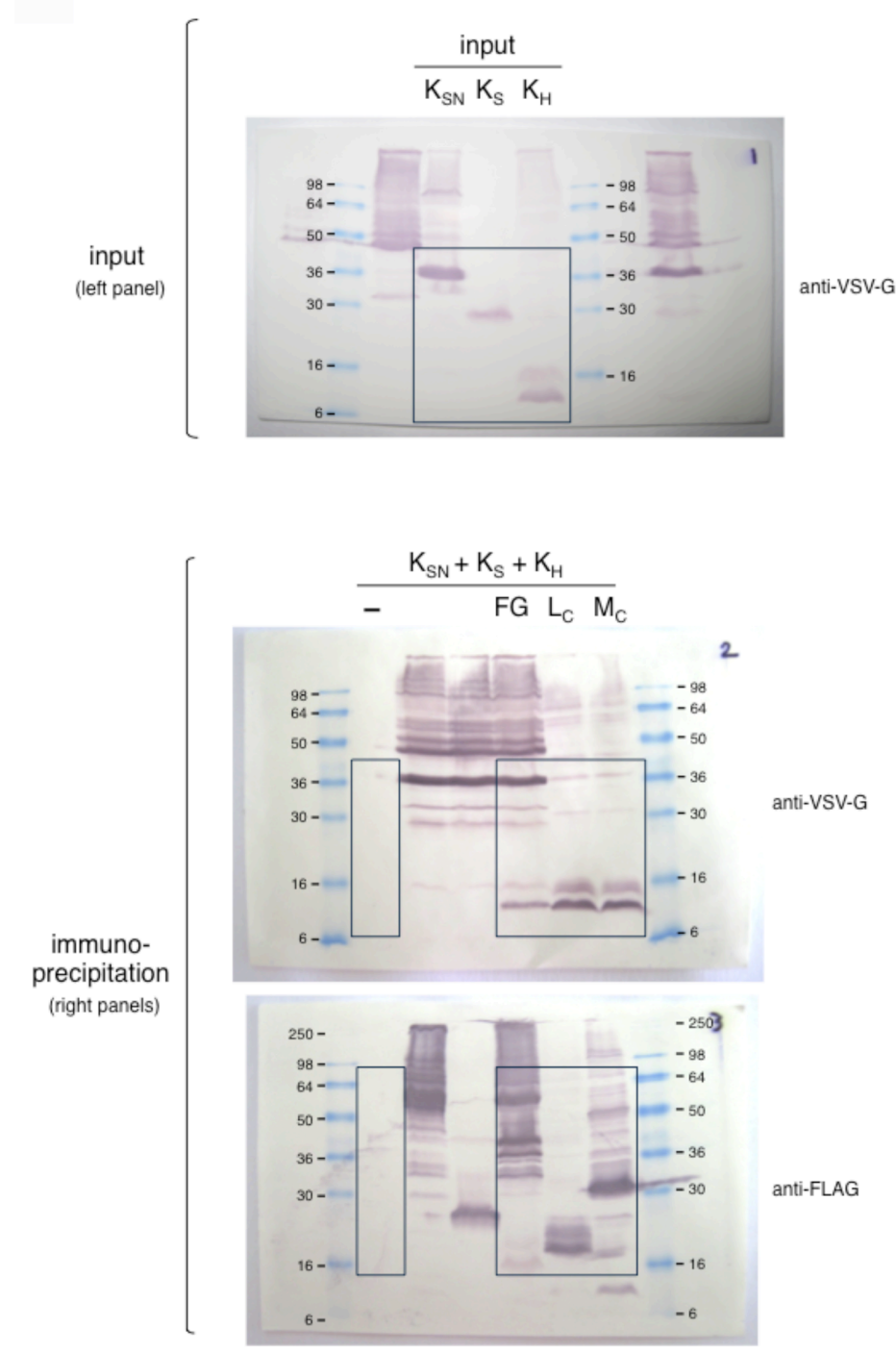

Supplementary Figure 10. Uncropped blots and gels.
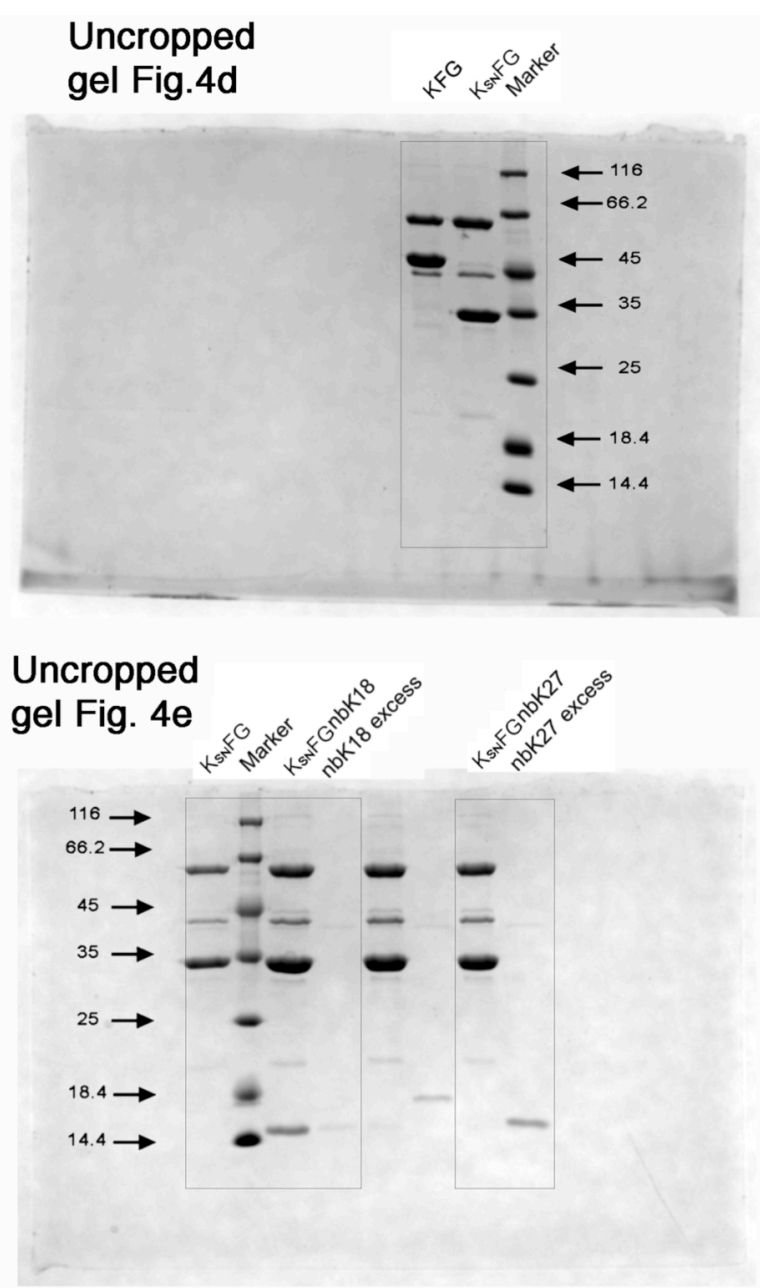
Supplementary Table 1. Data collection and refinement statistics of nbK18, nbK18-TssK and $\mathrm{TssK}_{\mathrm{SN}}-\mathrm{nbK} 18-\mathrm{nbK} 27$ complexes, and $\mathrm{TssK}_{\mathrm{H}}$ domain. (numbers in brackets refer to the highest resolution bin)

\begin{tabular}{|c|c|c|c|c|c|}
\hline DATA COLLECTION & nbK18 & $\begin{array}{c}\text { TssK-nbK18 } \\
\text { CsI/NaI }\end{array}$ & $\begin{array}{c}\text { TssK-nbK18 } \\
\text { native }\end{array}$ & $\begin{array}{c}\mathrm{TssK}_{\mathrm{SN}}-\mathrm{nbK} 18- \\
\text { nbK27 }\end{array}$ & $\mathrm{TssK}_{\mathrm{H}}$ \\
\hline PDB & $5 \mathrm{M} 2 \mathrm{~W}$ & & $5 \mathrm{M} 30$ & $5 \mathrm{MWN}$ & $5 \mathrm{M} 2 \mathrm{Y}$ \\
\hline Source & ESRF ID23-2 & Soleil PX 1 & Soleil PX 1 & ESRF ID30A-3 & ESRF ID23-1 \\
\hline Wavelength $(\AA)$ & 0.9786 & 1.7712 & 0.9786 & 0.9677 & 0.9789 \\
\hline Space group & $\mathrm{P} 4_{3}$ & $\mathrm{P} 22_{1} 2_{1}$ & $\mathrm{P} 22_{1} 2_{1}$ & $\mathrm{P} 22_{1} 2_{1}$ & $\mathrm{P} 3_{2}$ \\
\hline Cell (Å) & $\begin{aligned} \mathrm{a}=\mathrm{b} & =53.4 \AA, \mathrm{c} \\
& =88.0 \AA\end{aligned}$ & $\begin{array}{c}a=93.4, b=153.7 \\
c=154.2\end{array}$ & $\begin{array}{c}\mathrm{a}=93.2, \mathrm{~b}=153.7 \\
\mathrm{c}=154.8\end{array}$ & $\begin{array}{c}\mathrm{a}=90.9, \mathrm{~b}=143.3 \\
\mathrm{c}=150.3\end{array}$ & $\begin{array}{c}\mathrm{a}=\mathrm{b}=63.8 \\
\mathrm{c}=63.1\end{array}$ \\
\hline Angles $\left(^{\circ}\right)$ & $\alpha=\beta=\gamma=90$ & $\alpha=\beta=\gamma=90$ & $\alpha=\beta=\gamma=90$ & $\alpha=\beta=\gamma=90$ & $\alpha=\beta=90$ \\
\hline Nr. of monomers & 2 & $3 / 3$ & $3 / 3$ & $3 / 3 / 1$ & 2 \\
\hline Resolution limits $(\AA)$ & $\begin{array}{c}20-1.50 \\
(1.59-1.5)\end{array}$ & $\begin{array}{c}50-3.49 \\
(3.6-3.49)\end{array}$ & $\begin{array}{c}50.0-2.6 \\
(2.66-2.6)\end{array}$ & $\begin{array}{l}50.0-2.20 \\
(2.33-2.20)\end{array}$ & $\begin{array}{c}41.6-1.61 \\
(1.71-1.61)\end{array}$ \\
\hline Rmerge $\left(\right.$ Rpim $\left.^{\mathrm{a}}\right)$ & $0.088(0.785)$ & $0.016(1.8)$ & $0.018(1.40)^{\mathrm{a}}$ & $0.077(0.84)$ & $0.06(0.65)$ \\
\hline $\mathrm{CC} 1 / 2$ & $0.994(0.61)$ & $0.999(0.32)$ & $1.0(0.30)$ & $0.993(0.5)$ & $0.99(0.90)$ \\
\hline Unique reflections & $38858(6348)$ & 54138 & 69265 (4986) & $100494(16357)$ & $36972(5934)$ \\
\hline $\operatorname{Mean}((\mathrm{I}) / \operatorname{sd}(\mathrm{I}))$ & $7.8(1.6)$ & $14.6(1.0)$ & $19.6(0.5)$ & $6.5(1.0)$ & $22.79(3.36)$ \\
\hline Completeness (\%) & $98.7(98.2)$ & $100(99.5)$ & $99.8(99.2)$ & $95.5(92.4)$ & $99.9(99.5)$ \\
\hline Multiplicity & $3.5(3.3)$ & $18(8)$ & $11.0(10.7)$ & $4.8(4.4)$ & $10.6(10.5)$ \\
\hline SigAno $(4.18 \AA)$ & & $7.9(0.94)$ & & & \\
\hline CCano $(4.18 \AA)$ & & $0.98(0.14)$ & & & \\
\hline \multicolumn{6}{|l|}{ REFINEMENT } \\
\hline Resolution $(\AA)$ & $\begin{array}{c}12.1-1.5 \\
(1.54-1.5)\end{array}$ & & $\begin{array}{c}48.9-2.6 \\
(2.67-2.6)\end{array}$ & $\begin{array}{c}45.0-2.20 \\
(2.26-2.20)\end{array}$ & $\begin{array}{c}12.7-1.61 \\
(1.651 .61)\end{array}$ \\
\hline Number of reflections & $38782(2908)$ & & $59771(4503)$ & $98369(6904)$ & $36903(2686)$ \\
\hline $\begin{array}{l}\text { Number of protein / } \\
\text { water / ions atoms }\end{array}$ & $1712 / 296 / 15$ & & $10297 / 120$ & $10822 / 694$ & $1966 / 223$ \\
\hline Test set reflections & $1940(146)$ & & 2988 & 5032 & 1845 \\
\hline $\mathrm{R}_{\text {work }} / \mathrm{R}_{\text {free }}$ & $\begin{array}{c}0.195 / 0.212 \\
(0.3 / 0.28)\end{array}$ & & $\begin{array}{c}0.198 / 0.218 \\
(0.249 / 0.295)\end{array}$ & $\begin{array}{c}0.199 / 0.220 \\
(0.232 / 0.249)\end{array}$ & $\begin{array}{c}0.198 / 0.199 \\
(0.216 / 0.246)\end{array}$ \\
\hline $\begin{array}{l}\text { r.m.s.d.bonds } \\
(\AA) / \text { angles }\left(^{\circ}\right)\end{array}$ & $0.011 / 1.13$ & & $0.009 / 1.09$ & $0.008 / 1.07$ & $0.012 / 1.12$ \\
\hline B-Wilson / B-mean $\AA$ & $22.7 / 28.6$ & & $108.5 / 107.1$ & $57.1 / 66.4$ & $25.7 / 32.2$ \\
\hline $\begin{array}{c}\text { Ramachandran: } \\
\text { preferred / allowed / } \\
\text { outliers }(\%)\end{array}$ & $96.3 / 3.7 / 0$ & & $97.1 / 2.6 / 0.3$ & $79.9 / 2.1 / 0$ & $98.0 / 2.0 / 0$ \\
\hline
\end{tabular}


Supplementary Table 2. Interaction contacts between TssK and the nbK18 nanobody (obtained with the PISA server (Krissinel \& Henrick, 2007)). ASA: accessible surface area $\left(\AA^{2}\right)$. BSA: buried surface area $\left(\AA^{2}\right)$. a) surface of nbK18; blue: CDR1; green: CDR2; pink: CDR3. b) surface of TssK. (The bars indicate the percentage of the ASA covered; 1 bar $=10 \%$ ).

a)

\begin{tabular}{|c|c|c|c|}
\hline & H-bonds & ASA & BSA \\
\hline VAL 32 & & 56.25 & 42.21|||||||| $\mid$ \\
\hline VAL 33 & & 37.25 & 32.81|||||||||| \\
\hline ALA 35 & & 8.52 & 8.37\|\|\|\|\|\|\|\| \\
\hline TYR 37 & $\mathrm{H}$ & 41.54 & 25.97\|\|\|\| \\
\hline total & & & 125 \\
\hline LEU 47 & & 79.47 & $60.17 \quad|||||||| \mid$ \\
\hline SER 50 & & 18.33 & 10.61\|\|\|\| \\
\hline THR 52 & $\mathrm{H}$ & 49.89 & $18.84|\|| \mid$ \\
\hline GLY 54 & & 75.43 & $13.20 \|$ \\
\hline THR 56 & $\mathrm{H}$ & 86.79 & 9.83 \\
\hline ALA 60 & & 21.75 & 13.55\|\|\|\|$\|$ \\
\hline total & & & 132 \\
\hline LYS 96 & $\mathrm{H}$ & 33.05 & $22.99 \quad\|\|\|\| \|$ \\
\hline PHE 98 & & 73.41 & $72.20 \quad|||||||||| \mid$ \\
\hline ARG 100 & $\mathrm{H}$ & 133.07 & 69.68\|\|\|\|$\|$ \\
\hline TYR 103 & & 188.69 & 110.41\|\|\|\| $\mid$ \\
\hline VAL 104 & & 79.31 & $32.53 \mathrm{\| U \|}$ \\
\hline GLY 105 & $\mathrm{H}$ & 38.52 & $38.52\||\||||||||$ \\
\hline TYR 106 & & 89.32 & $33.59 \quad|\||$ \\
\hline ASP 107 & $\mathrm{H}$ & 79.14 & 65.47|||||||||| \\
\hline total & & & 452 \\
\hline Grand total & & & 709 \\
\hline
\end{tabular}

b)

\begin{tabular}{|c|c|c|c|}
\hline & H-bonds & ASA & BSA \\
\hline PRO 69 & & 47.50 & 37.02 ШШШ \\
\hline ASP 70 & & 44.65 & $21.34 \mathrm{WW}$ \\
\hline LEU 105 & & 78.77 & 78.45 ШШШШШ \\
\hline LEU 106 & $\mathrm{H}$ & 70.66 & 33.60 ШШ \\
\hline ASN 107 & $\mathrm{H}$ & 42.33 & 36.39 \\
\hline ALA 108 & $\mathrm{H}$ & 106.15 & $35.88 \mathrm{UW}$ \\
\hline ASN 109 & $\mathrm{H}$ & 149.19 & 58.67 Ш \\
\hline GLY 110 & & 39.76 & $11.02 \amalg$ \\
\hline GLU 118 & & 170.72 & $61.86 \mathrm{WW}$ \\
\hline SER 119 & & 48.16 & $17.95 \amalg \amalg$ \\
\hline GLU 120 & $\mathrm{H}$ & 165.08 & 139.61 \\
\hline ARG 121 & & 54.64 & 43.11 ШШШШ \\
\hline GLU 158 & $\mathrm{H}$ & 160.85 & 84.97 لШШ ل \\
\hline ALA 160 & $\mathrm{H}$ & 97.20 & $48.41 \mathrm{WW}$ \\
\hline ALA 161 & & 35.99 & 24.80 ШШШШ \\
\hline TRP 162 & $\mathrm{H}$ & 48.61 & $8.76 \Perp$ \\
\hline LEU 163 & & 22.45 & 21.13 \\
\hline
\end{tabular}


Supplementary Table 3. Strains, plasmids and oligonucleotides used in this study.

\section{Strains}

Strains

Description and genotype

Source

\section{E. coli $\mathrm{K}-12$}

DH5 $\alpha$

W3110

BTH101

T7 Iq pLys
F-, $\Delta(\operatorname{argF-lac}) \mathrm{U} 169$, phoA, supE44, $\Delta(\mathrm{lacZ}) \mathrm{M} 15$, relA, endA, thi, hsdR

F-, lambda- IN(rrnD-rrnE) 1 rph-1

F-, cya-99, araD139, galE15, galK16, rpsL1 (Str r), hsdR2, mcrA1, mcrB1.

MiniF, lys $Y$, lacl $^{q}\left(\mathrm{Cam}^{\mathrm{R}}\right)$, fhuA2, lacZ::T7 gene1, ompT, gal, sulA11,

$R\left(m c r-73: \because\right.$ miniTn10--Tet $\left.{ }^{\mathrm{S}}\right) 2, d c m, R\left(z g b-210: \because\right.$ Tn10--Tet $\left.{ }^{\mathrm{S}}\right)$ endA1 $\Delta(m c r C-m r r)$
New England Biolabs

Laboratory collection

Karimova et al., 2005

New England Biolabs

Enteroaggregative E. coli

$17-2$

WT enteroaggregative Escherichia coli
Arlette Darfeuille-Michaud 


\section{Plasmids}

\section{Protein production vectors}

pRSF-1

pRSF-TssK 6 His

pETG20A

pETG-TssK ${ }_{\mathrm{Ct}}$ cloning vector, $\mathrm{P} T 7, \mathrm{Kan}^{\mathrm{R}}$

sci-1 tssK cloned into pRSF-1, C-terminal $6 \times$ His sequence

Gateway ${ }^{\circledR}$ destination vector, $6 \times$ His-TRX followed by a TEV cleavage site sci-1 tssK residues 316-445 cloned into pETG20A
Novagen

This study Arie Geerlof This study

\section{Expression vectors}

pASK-IBA37

pASK-IBA37-Tss FLAG $_{\text {FL }}$

pASK-IBA37-TssF FLAG

pASK-IBA37-TssG GLAG

pASK-IBA37-fLAGTssLc pASK-IBA37-TssM $\mathrm{C}_{\mathrm{C}} \mathrm{NTP}_{\mathrm{FLAG}}$ pBAD33

pBAD33-TssK $\mathrm{VSV}_{\text {-G }}$ pBAD33-TssK-SN ${ }_{V S V-G}$ pBAD33-TssK-H ${ }_{V s v-G}$ pBAD33-TssK-SVsv-G pBAD33-TssK-N ${ }_{\text {Vsv-G }}$ pUC-Hcp $F L A G$ cloning vector, Ptet, $\mathrm{Amp}^{\mathrm{R}}$

sci-1 tss $A$ cloned into pASK-IBA37, C-terminal FLAG epitope sci-1 tss $F$ cloned into pASK-IBA37, C-terminal FLAG epitope $s c i-1$ tss $G$ cloned into pASK-IBA37, C-terminal FLAG epitope sci-1 tss $L$ residues 1-184 cloned into pASK-IBA37, N-terminal FLAG epitope sci-1 tss $M$ residues 62-273 cloned into pASK-IBA37, C-terminal FLAG epitope cloning vector, pACYC184 origin, Para, araC, $\mathrm{Cm}^{\mathrm{R}}$ sci-1 tss $K$ cloned into pBAD33, C-terminal VSV-G epitope sci-1 tss $K$ residues 1-315 cloned into pBAD33, C-terminal VSV-G epitope sci-1 tssK residues 315-445 cloned into pBAD33, C-terminal VSV-G epitope sci-1 tssK residues 1-193 cloned into pBAD33, C-terminal VSV-G epitope sci-1 tssK residues 175-315 cloned into pBAD33, C-terminal VSV-G epitope sci-1 hcp cloned into pUC12, Plac, C-terminal FLAG epitope
IBA Technology Zoued et al., 2016 Brunet et al., 2015

Brunet et al., 2015 Aschtgen et al., 2012 Logger et al., 2016 Guzman et al., 1995 Brunet et al., 2015 This study This study This study This study Aschtgen et al., 2008 


\begin{tabular}{|c|c|c|}
\hline pT18-FLAG & $\begin{array}{l}\text { Bacterial Two Hybrid vector, ColE1 origin, Plac, T18 fragment of Bordetella } \\
\text { pertussis CyaA, Amp }^{\mathrm{R}}\end{array}$ & Battesti \& Bouveret, 2008 \\
\hline pTssK-T18 & tss $K$ cloned upstream T18 in pT18-FLAG & Zoued et al., 2013 \\
\hline pTssK $_{\mathrm{S}}-\mathrm{T} 18$ & tss $K$ residues $1-193$ cloned upstream T18 in pT18-FLAG & This study \\
\hline $\mathrm{pT} 18-\mathrm{TssK}_{\mathrm{N}}$ & tss $K$ residues $175-315$ cloned downstream T18 into pT18-FLAG & This study \\
\hline $\mathrm{pTssK}_{\mathrm{SN}} \mathrm{T}^{-\mathrm{T}}$ & tss $K$ residues $1-315$ cloned upstream T18 into pT18-FLAG & This study \\
\hline pT18-TssK ${ }_{H}$ & tss $K$ residues $315-445$ cloned downstream T18 into pT18-FLAG & This study \\
\hline pT18-Pal & pal cloned downstream the T18 coding sequence in pT18-FLAG & Battesti \& Bouveret, 2008 \\
\hline pT25-FLAG & $\begin{array}{l}\text { Bacterial Two Hybrid vector, p15A origin, Plac, T25 fragment of Bordetella } \\
\text { pertussis CyaA, Kan }{ }^{\mathrm{R}}\end{array}$ & Battesti \& Bouveret, 2008 \\
\hline pT25-TssFG & tss $F G$ cloned downstream the T25 coding sequence in pT25-FLAG & Brunet et al., 2015 \\
\hline pT25-TssK & tss $K$ cloned downstream the T25 coding sequence in pT25-FLAG & Zoued et al., 2013 \\
\hline pTssL $_{C}-\mathrm{T} 25$ & tss $L$ residues $1-184$ cloned downstream the T25 coding sequence in pT25-FLAG & Durand et al., 2012 \\
\hline $\mathrm{pTssM}_{\mathrm{C}}-\mathrm{T} 25$ & tss $M$ residues $62-360$ cloned upstream the T25 coding sequence in pT25-FLAG & Zoued et al., 2013 \\
\hline pT25-TolB & tolB cloned upstream the T25 coding sequence in pT25-FLAG & Battesti \& Bouveret, 2008 \\
\hline
\end{tabular}




\section{Oligonucleotides}

\section{Name}

Destination

Sequence $\left(5^{\prime} \rightarrow 3^{\prime}\right)$

For plasmid construction $^{\text {b,c,d }}$

5- pBAD33-TssK-SN $N_{V S V-G}$

3- pBAD33-TssK-SN ${ }_{\text {VSV-G }}$

5- pBAD33-TssK-HVsv-G

3- pBAD33-TssK-HVsV-G

3-pBAD33-TssK-S

5-pBAD33-TssK-N

$$
\text { insertion of } t s s K_{175-315} \text { fragment into pBAD33 }
$$

5-pTssK $-\mathrm{T} 18$

$3-\mathrm{pTssK} \mathrm{S}_{\mathrm{S}} \mathrm{T} 18$

5-pT18-TssK $\mathrm{N}_{\mathrm{N}}$

3-pT18-TssK ${ }_{N}$ insertion of $t s s K_{1-193}$ fragment into pT18-Flag insertion of $t s s K_{1-193}$ fragment into pT18-Flag insertion of $t s s K_{175-315}$ fragment into pT18-Flag

insertion of $t s s K_{1-315}$ fragment into $\mathrm{pBAD} 33$

insertion of $t s s K_{1-315}$ fragment into pBAD33

insertion of $t s s K_{315-445}$ fragment into pBAD33

insertion of $t s s K_{315-445}$ fragment into pBAD33

insertion of $t s s K_{1-193}$ fragment into pBAD33

insertion of $t s s K_{175-315}$ fragment into pT18-Flag
CTCTCTACTGTTTCTCCATACCCGTTTTTTTGGGCT AGCAGGAGGTATTACACCATGAAGATTTATCGCCCA TTATGGGAAGACG

GGTCGACTCTAGAGGATCCCCGGGTACCTTATTT TCCTAATCTATTCATTTCAATATCTGTATAGGAAGG CAGGCTTGCCTCCAGC CTCTCTACTGTTTCTCCATACCCGTTTTTTTGGGCT AGCAGGAGGTATTACACCATGCGTGTGG

TTTTCATTGAACTGAAACAAAAGGC GGTCGACTCTAGAGGATCCCCGGGTACCTTATTT TCCTAATCTATTCATTTCAATATCTGTATATGT CCGCAGCACCGCAAAAAGTTC

GGTCGACTCTAGAGGATCCCCGGGTACCTTA TTTTCCTAATCTATTCATTTCAATATCTGTATA GGCAGACAGCGTCAGCAGAGGG CTCTCTACTGTTTCTCCATACCCGTTTTTTTGGGCT AGCAGGAGGTATTACACCATGGGACAGTGG TGCAGGGACCCGCG

CGGATAACAATTTCACACAGGAAACAGCTATGACC ATGAAGATTTATCGCCCATTATGGGAAGACG CCTCGCTGGCGGCTAAGCTTGGCGTAAT GGCAGACAGCGTCAGCAGAGGG GCCACTGCAGGGATTATAAAGATGACGATGA CAAGGGACAGTGGTGCAGGGACCCGC AGGTCGACGGTATCGATAAGCTTGATATCGAATTC 
TAGTTAGGAAGGCAGGCTTGCCTCCAGC

$5-\mathrm{p} \operatorname{TssK}_{\mathrm{SN}}-\mathrm{T} 18$ insertion of $t s s K_{1-315}$ fragment into pT18-Flag

$3-\mathrm{TssK}_{\mathrm{SN}} \mathrm{T} 18$

5-pT18-TssK $\mathrm{H}_{\mathrm{H}}$

3-pT18-TssK $\mathrm{H}_{\mathrm{H}}$

T6_K316-445_p17_F

T6_K316-445_p17_R insertion of $t s s K_{1-315}$ fragment into pT18-Flag

insertion of $t s s K_{315-445}$ fragment into pT18-Flag

insertion of $t s s K_{315-445}$ fragment into pT18-Flag

insertion of $t s s K_{316-445}$ fragment into pETG20A

insertion of $t s s K_{316-445}$ fragment into pETG20A
CGGATAACAATTTCACACAGGAAACAGCTATGACC ATGAAGATTTATCGCCCATTATGGGAAGACG CCTCGCTGGCGGCTAAGCTTGGCGTAATGGAAG GCAGGCTTGCCTCCAGC CGCCACTGCAGGGATTATAAAGATGACGATGACA AGCGTGTGGTTTTCATTGAACTGAAACAAAAGGG CGAGGTCGACGGTATCGATAAGCTTGATATCGAA TTCTAGTTATGTCCGCAGCACCGCAAAAAGTTC GGCTTAGAAAACCTGTACTTCCAGGGTTCCCGT

GTGGTTTTCATTGAACTGAAAC

TTGTACAAGAAAGCTGGGTTTATTATGTCC

GCAGCACCGCAAAAAGTTCC

${ }^{a}$ Sequence annealing on the target plasmid underlined.

${ }^{\mathrm{b}}$ VSV-G epitope coding sequence italicized. 